\title{
Epigenetic Mechanisms Mediating Cell State Transitions in Chondrocytes
}

\author{
Manuela Wuelling, ${ }^{1 \dagger}$ (1) Christoph Neu, ${ }^{1 \dagger}$ (D) Andrea M Thiesen, ${ }^{1}$ Simo Kitanovski, ${ }^{2}$ (D) Yingying Cao, $^{2}$ \\ Anja Lange, ${ }^{2}$ Astrid M Westendorf, ${ }^{3}$ (i) Daniel Hoffmann, ${ }^{2}$ (i) and Andrea Vortkamp ${ }^{1}$ (i) \\ ${ }^{1}$ Developmental Biology, Centre for Medical Biotechnology, University Duisburg-Essen, Essen, Germany \\ ${ }^{2}$ Bioinformatics and Computational Biophysics, Centre for Medical Biotechnology, University Duisburg-Essen, Essen, Germany \\ ${ }^{3}$ Institute of Medical Microbiology, University Hospital Essen, University Duisburg-Essen, Essen, Germany
}

\begin{abstract}
Epigenetic modifications play critical roles in regulating cell lineage differentiation, but the epigenetic mechanisms guiding specific differentiation steps within a cell lineage have rarely been investigated. To decipher such mechanisms, we used the defined transition from proliferating (PC) into hypertrophic chondrocytes $(\mathrm{HC})$ during endochondral ossification as a model. We established a map of activating and repressive histone modifications for each cell type. ChromHMM state transition analysis and Pareto-based integration of differential levels of mRNA and epigenetic marks revealed that differentiation-associated gene repression is initiated by the addition of H3K27me3 to promoters still carrying substantial levels of activating marks. Moreover, the integrative analysis identified genes specifically expressed in cells undergoing the transition into hypertrophy. Investigation of enhancer profiles detected surprising differences in enhancer number, location, and transcription factor binding sites between the two closely related cell types. Furthermore, cell type-specific upregulation of gene expression was associated with increased numbers of H3K27ac peaks. Pathway analysis identified PC-specific enhancers associated with chondrogenic genes, whereas HC-specific enhancers mainly control metabolic pathways linking epigenetic signature to biological functions. Since HC-specific enhancers show a higher conservation in postnatal tissues, the switch to metabolic pathways seems to be a hallmark of differentiated tissues. Surprisingly, the analysis of H3K27ac levels at super-enhancers revealed a rapid adaption of H3K27ac occupancy to changes in gene expression, supporting the importance of enhancer modulation for acute alterations in gene expression. $\odot 2021$ The Authors. Journal of Bone and Mineral Research published by Wiley Periodicals LLC on behalf of American Society for Bone and Mineral Research (ASBMR).
\end{abstract}

KEY WORDS: EPIGENETICS; GENETIC RESEARCH; CHONDROCYTE AND CARTILAGE BIOLOGY; GROWTH PLATE; CELL/TISSUE SIGNALING; TRANSCRIPTION FACTORS; DEVELOPMENTAL MODELING; BONE MODELING AND REMODELING

\section{Introduction}

$\mathrm{n}$ the last decade, the role of epigenetic modifications in regulating
tissue differentiation and development has been intensively studied.
Most of these studies have focused on the differentiation of distinct cell
lineages from embryonic and mesenchymal stem cells, or the compar-
ison of distinct tissues, ${ }^{(1-3)}$ but the epigenetic modifications regulating
changes in gene expression at discrete differentiation steps within a
specific cell lineage are less well understood. To receive insight into
such epigenetic mechanisms, we have investigated the transition of
proliferating into hypertrophic chondrocytes during endochondral
ossification as a representative differentiation step. The switch between
the two differentiation states takes place at a precise morphological
position and is tightly controlled by numerous transcription factors, signaling pathways, and epigenetic modifiers, ${ }^{(4-7)}$ allowing us to relate differences in the epigenetic profile to gene function.

Endochondral ossification is initiated by the formation of Sox9 and Collagen type 2a1 (Col2a1) expressing condensations of proliferating chondrocytes (PC). Once these templates of the later bones have reached a critical size, PC in their center exit the cell cycle and differentiate into hypertrophic chondrocytes $(\mathrm{HC})$, which express Collagen type 10a1 (Col10a1) instead of Col2a1 and mineralize their extracellular matrix (ECM). Because $\mathrm{HC}$ are subsequently replaced by ossified bone tissue, the transition from $\mathrm{PC}$ to $\mathrm{HC}$ is critical in balancing growth and stability of the later skeletal elements.

Epigenetic modifications relevant for the formation of distinct tissues include the acetylation and methylation of specific histone

This is an open access article under the terms of the Creative Commons Attribution-NonCommercial-NoDerivs License, which permits use and distribution in any medium, provided the original work is properly cited, the use is non-commercial and no modifications or adaptations are made.

Received in original form October 28, 2020; revised form January 21, 2021; accepted January 26, 2021; Accepted manuscript online February 3, 2021.

Address correspondence to: Andrea Vortkamp, PhD, Developmental Biology, University Duisburg-Essen, Universitaetsstrasse 2, 45141 Essen, Germany. E-mail: andrea.vortkamp@uni-due.de

Additional Supporting Information may be found in the online version of this article.

${ }^{\dagger} \mathrm{MW}$ and $\mathrm{CN}$ contributed equally to this work.

Journal of Bone and Mineral Research, Vol. 36, No. 5, May 2021, pp 968-985.

DOI: 10.1002/jbmr.4263

( 2021 The Authors. Journal of Bone and Mineral Research published by Wiley Periodicals LLC on behalf of American Society for Bone and Mineral Research (ASBMR). 
residues. Whereas histone acetylation results in an opening of the chromatin structure and, consequently, the activation of gene expression, histone deacetylation leads to a dense chromatin structure and gene silencing. Acetylated histones are localized at distinct regulatory regions, as, for example, acetylated lysine 9 of histone 3 (H3K9ac), which demarcates active promoters, whereas acetylated lysine 27 (H3K27ac) marks active promoters and enhancers. In contrast to acetylation, histone methylation elicits positive or negative effects on gene expression depending on the position of the methylated residue. Trimethylation of lysine 4 of histone 3 (H3K4me3) defines the promoter of active genes and genes primed for expression ${ }^{(8)}$ and trimethylation of lysine 36 of histone $\mathrm{H} 3(\mathrm{H} 3 \mathrm{~K} 36 \mathrm{me})$ is associated with actively transcribed genes. ${ }^{(9)}$ In contrast, trimethylation of histone $\mathrm{H} 3$ at lysine 9 (H3K9me3) or lysine 27 (H3K27me3) leads to gene repression. ${ }^{(10,11)}$ Besides different combinations of activating marks at promoter regions, the combination of activating $\mathrm{H} 3 \mathrm{~K} 4 \mathrm{me} 3$ and repressive $\mathrm{H} 3 \mathrm{~K} 27 \mathrm{me} 3$, the so-called bivalent mark, is found at promoters of many lineage-specific genes in embryonic stem cells (ESCs) and has been associated with pluripotency. ${ }^{(12)}$ During differentiation, genes carrying this bivalent mark can either gain additional activating histone marks and lose $\mathrm{H} 3 \mathrm{~K} 27 \mathrm{me} 3$ to initiate transcription or lose H3K4me3, leading to gene repression. ${ }^{(13,14)}$ Recently, another bivalent chromatin state, a combination of $\mathrm{H} 3 \mathrm{~K} 36 \mathrm{me} 3$ and H3K9me3, has been described on weakly transcribed genes. ${ }^{(15,16)}$

In chondrocyte, initial studies investigated epigenetic marks associated with binding of the transcription factor Sox9 in postnatal rib chondrocytes ${ }^{(17)}$ and rat chondrosarcoma cells. ${ }^{(18)}$ More recently, the analysis of open chromatin in mixed growth plate chondrocytes by ATAC-seq identified specific enhancers modulating skeletal height ${ }^{(19)}$ and osteoarthrosis susceptibility, ${ }^{(20)}$ but a systematic investigation of the epigenetic mechanisms controlling distinct steps of chondrocyte differentiation in vivo is still lacking.

In this study, we have analyzed the epigenetic alterations regulating the cell state transition from $\mathrm{PC}$ to $\mathrm{HC}$, and correlated them to differences in gene expression. By establishing an unbiased epigenetic profile of activating and repressive histone modifications, we identified the addition of $\mathrm{H} 3 \mathrm{~K} 27 \mathrm{me} 3$ to promoter regions still carrying activating marks as the critical event to induce the repression of genes downregulated with the switch into hypertrophy. Pareto-based integration of differential levels of activating and repressive histone marks supported this hypothesis. It further identified a set of genes specifically upregulated in prehypertrophic chondrocytes, including new potential regulators of the onset of hypertrophy. Analysis of H3K27ac occupancy at enhancers revealed an unexpected difference in enhancer usage between the closely related cell types. Moreover, while PC-specific enhancers regulated mainly genes associated with chondrogenesis, HC-specific enhancers were primarily associated with metabolic pathways identifying metabolism as a main biological function of hypertrophic chondrocytes. The higher conservation of HC-specific enhancers in postnatal tissues indicates that the switch to metabolic pathways seems to be a hallmark of differentiated tissues. Investigating the H3K27ac levels at superenhancers (SE), we found rapid changes in H3K27ac occupancy related to altered gene expression, supporting the importance of enhancer modulation for acute alterations in gene expression.

\section{Materials and Methods}

Animal models

Collagen 2a1-Cre (Tg[Col2a1-cre]1Star $\left.{ }^{(21)}\right)$, Collagen 10-Cre mice (Tg [Col10a1-cre]1427Vdm), ${ }^{(22)}$ and ROSA26-YFP (yellow fluorescent protein) reporter (R26R-YFP, Gt[ROSA]26Sortm1[EYFP]Cos ${ }^{(23)}$ ) mice were maintained on a $\mathrm{C} 57 \mathrm{BI} / 6 \mathrm{~J}$ genetic background and crossed to generate timed pregnancies of Col2-Cre;R26RYFP and Col10Cre;R26R-YFP mice. Mouse husbandry was approved by the city of Essen (Az.32-2-11-80-71/203) in accordance with $\$ 11,1$ 1a of the "Tierschutzgesetz" and approved by the animal welfare committee of the University Duisburg-Essen.

\section{Cell sorting and chromatin immunoprecipitation with next-generation sequencing (ChIP-seq)}

For chondrocyte isolation, YFP-positive embryos were decapitated. The axial and appendicular skeletons were freed from skin, muscle, and internal organs under a dissecting microscope (Leica MZ5, Wetzlar, Germany), dissociated with $0.6 \mathrm{U} / \mathrm{mL}$ collagenase (Serva Electrophoresis, Heidelberg, Germany) and 0.25 trypsin (Thermo Fisher Scientific, Dreieeichen, Germany), filtered through a $70 \mu \mathrm{m}$ cell strainer (Falcon) and fixed with $1 \%$ formaldehyde (Applichem, Darmstadt, Germany) before cell sorting to eliminate potential fluorescence-activated cell sorting (FACS)induced stress responses and corresponding alterations in chromatin structure. PC and HC were isolated from E13.5 Col2-Cre; R26R-YFP and E15.5 Col10-Cre;R26R-YFP embryos, respectively, by FACS of YFP-positive cells using a FACSAriall cell sorter (BD Biosciences, Heidelberg, Germany) with a $70 \mu \mathrm{m}$ nozzle. Cells were enriched to a purity of $77.6 \%$ to $96.4 \%$ for PC and $72.0 \%$ to $98.5 \%$ for $\mathrm{HC}$, measured in the reanalysis. To reduce biological variation, cells of different sorts were pooled before chromatin immunoprecipitation (ChIP). A total of $3.5 \times 10^{5}$ cells were used for each ChIP. Chromatin was sonicated for 30 minutes using a Biorupter (Diagenode, Denville, NJ, USA) and isolated using the True MicroChip Kit (Diagenode). Antibodies were obtained from Diagenode (H3K4me3, H3K36me3, H3K9me3, H3K27me3, lgG) or Abcam (H3K9ac, H3K27ac; Cambridge, UK). Library preparation was performed with the MicroPlex Library preparation Kit V2 (Diagenode) and $61 \mathrm{bp}$ reads were sequenced on a Hiseq 2500 (Illumina, San Diego, CA, USA) at a depth of 20 to 30 million reads per experiment. ChIP was performed in biological triplicate for PC, each replicate representing cells of approximately 10 embryos, and in duplicate for $\mathrm{HC}$, including cells of approximately 50 embryos per replicate.

\section{ChIP-seq data processing}

Read quality of the raw sequences was verified with fastqc (version $\left.0.11 .9^{(24)}\right)$. All reads were trimmed to a length of 60 bases and those with a mean Phread score below 20 were discarded using prinseqlite (version $0.20 .4^{(25)}$ ). The remaining reads were mapped to the mm10 build (UCSC mm10, Dec. 2011) of the mouse reference genome with bwa (version 0.7.15 ${ }^{(26)}$ ) using default setting, except the maximum edit distance, which was set to 4. Unmapped reads and reads with a mapping quality below 30 were removed with samtools (version 1.4/1.5 ${ }^{(26)}$ ). Duplicate reads were filtered with Picard tools (version 2.9, http://broadinstitute.github.io/ picard/). Mapping quality statistics were computed using qualimap (version $2.1^{(27,28)}$ ). To assess the library complexity, the nonredundant fraction (NRF) and $\mathrm{PCR}$ bottlenecking coefficients (PBC, https://github.com/imbforge/encodeChIPqc/blob/master/ $\mathrm{R} / \mathrm{PBC}$.R) were calculated with functions implemented in $\mathrm{R}$ (version 3.6.1). Relative strand correlation (RSC) and normalized strand correlation (NSC), which indicate efficiency of the ChIP, were determined using Phantompeakqualtool ${ }^{(27,29)}$ (https://github.com/ kundajelab/phantompeakqualtools). Based on the FRiP (fraction 
of reads in peaks) parameter, the fraction of reads in enriched regions was calculated ${ }^{(29)}$ (FRIER, https://github.com/ BioinformaticsBiophysicsUDE/ChIPSeqQualityMetrics/blob/ master/FRIER.R). Briefly, the coverage fold-change over the global background in 3000 bp windows was computed in an interval from 0.1 to 4.0 using R-packages csaw and GenomicAlignment. ${ }^{(30,31)}$ The concordance between biological replicates was computed as Pearson and Spearman correlation (https://github. com/BioinformaticsBiophysicsUDE/ChIPSeqQualityMetrics/blob/ master/Spearman_Pearson_Correlation.R). For the mapped and filtered reads, the coverage was binned ( $5 \mathrm{~kb}$ bins) and the correlation was calculated with R. Quality parameters were set to match the criteria defined by the Encode consortium. The code is available at: https://github.com/BioinformaticsBiophysicsUDE/ ChIPSeqProcessingOld.

Peaks were identified with hiddenDomains (version $3.0^{(32)}$ ). As a control data set, all lgG data for one cell type were merged. The bin size was adjusted to $200 \mathrm{bp}$ (narrow domains for H3K4me3, H3K9ac, H3K27ac) or 800 bp (broad domains for H3K36me3, $\mathrm{H} 3 \mathrm{~K} 9 \mathrm{me} 3, \mathrm{H} 3 \mathrm{~K} 27 \mathrm{me} 3)$. The parameter for the maximum read count was decreased to 30 and the posterior probability threshold set to 0.6. Using a sliding window approach, peaks separated by one or two bins, for broad and narrow domains, respectively, were merged. Consensus peaks were obtained by taking the intersection of the biological replicates. Comparability between the different replicate numbers was tested by calculating the peak number and coverage for subsets of two PC replicates. The code is available at https://github.com/BioinformaticsBiophysicsUDE/ ChIPSeqPeakCalling. The Integrative Genomics Viewer (IGV, $\mathrm{http} / / /$ software.broadinstitute.org/software/igv ${ }^{(33)}$ ) was used to visualize histone marks and peaks at selected genomic locations. All sequencing and peak calling data are available at the GEO database (GSE155401).

\section{Defining epigenetic states with ChromHMM}

The peaks generated by hiddenDomains were used as input for ChromHMM (version 1.20 (34)). Each chromosome was segmented into non-overlapping bins of $200 \mathrm{bp}$. For each histone mark, a bin was set to 1 if it partially or completely overlapped with a peak, and to 0 in case of no overlap, generating a separate track of bins for each histone mark. This data set was used to train multiple ChromHMM models with increasing number of states. Based on their log-likelihoods and the biological interpretation, the 15-state ChromHMM model was selected. To assess the robustness of the model, the duplicate subsets of the PC and $\mathrm{HC}$ data sets were evaluated. Additionally, the Encode mouse reference epigenomes for limb (ENCSR283NCE, ENCSR589KOM, ENCSR424HQH, ENCSR705YBY, ENCSR486MYL) and forebrain (ENCSR415TUB, ENCSR296YDZ, ENCSR911FIM, ENCSR894URD, ENCSR438IXZ) from developmental stages E11.5 to E15.5 were included. The code is available at https:// github.com/snaketron/SourceChromHmm/code.zip.

\section{Genomic enrichment of ChromHMM states}

The enrichment of ChromHMM states was quantified for 10 functional annotations: RefSeq transcription start site (TSS), transcription end site (TES), regions within $2 \mathrm{~kb}$ around the TSS, exons, genes, CpG islands, VISTA (www.enhancer.lbl.gov; accessed 2018), Fantom enhancers (https://fantom.gsc.riken.jp; version 2015) and Zinc finger genes (ENSEMBL annotation for the keyword "zinc finger protein"), "all enhancers" describes Fantom enhancers present in at least one tissue, and "forelimb" are Fantom enhancers detected in forelimb only. Enhancer coordinates were converted to $\mathrm{mm} 10$ with liftover (https://genome.ucsc. edu/cgi-bin/hgLiftOver). For each ChromHMM state, the bin was set to 1 if it partially or completely overlapped with the genomic region assigned to the functional annotations. In case of no overlap, the bin was set to 0 . The enrichment of a state at a given annotation compared with the background was computed as $E_{i j}=\left(F_{i j} / F_{i}\right) /\left(F_{j} / F\right)$; where $i$ and $j$ index different ChromHMM states and functional annotations; $F$ represents the frequency of bins. ${ }^{(30)}$

\section{Enrichment analysis of ChromHMM state transitions}

For ChromHMM state transition analysis of $\mathrm{PC}$ to $\mathrm{HC}$, genes of the Gene Ontology (GO) terms, chondrocyte differentiation (GO:0002062), limb development (GO:0060173), and cartilage development (GO:0051216) (334 genes, Supplemental Table S2) and, as a control, a group of housekeeping genes (265 genes, ${ }^{(35)}$ Supplemental Table S2) were selected. For these genes, the occurrence of a given ChomHMM transition was compared with the occurrence in the full set of genes. From these data, a binomial regression model was fitted in a Bayesian framework (brms [2.10.0] to determine an enrichment or depletion of a transition in a given set of genes with default priors ${ }^{(36)}$ and rstan [2.19.2]) (Stan Development Team. 2018. RStan: the R interface to Stan. R package version 2.17.3. http://mc-stan.org).

$$
\begin{gathered}
\mathrm{Y}_{\mathrm{t}, \mathrm{x}} \sim \operatorname{\operatorname {dinomial}}\left(\theta_{\mathrm{t}, \mathrm{x}}, \mathrm{N}_{\mathrm{x}}\right) \\
\theta_{\mathrm{t}, \mathrm{x}}=\operatorname{logistic}\left(\alpha_{\mathrm{t}}+\beta_{\mathrm{t}} * \mathrm{X}_{\mathrm{t}, \mathrm{x}}\right)
\end{gathered}
$$

The number of genes with transition $t$ and the condition $\mathrm{x}$ (part of the background $[\mathrm{x}=0$ ] or the selected group $[x=1])$ is $Y_{t, x}$. It depends on the number of genes in condition $x\left(\mathrm{~N}_{\mathrm{x}}\right)$ and the probability $\theta_{\mathrm{t}, \mathrm{x}}$ that a given transition $t$ occurs in a gene that is annotated to one of the two conditions $x$. The influence of the condition on $\theta$ is expressed by the effect size $\beta$, which is the log odds ratio. The uncertainty that an enrichment (positive log odds ratio) or a depletion (negative log odds ratio) occurs is represented by the probability that $\beta$ is either negative or positive, respectively. The accuracy of the model was tested by a posterior predictive check and the ability to recover simulated data. The code is available at https://github.com/ keksundso/StateTransitionEnrichment.

\section{Enhancer analysis}

The consensus H3K27ac peaks, separated into common, PC-specific, and HC-specific peaks, were compared with reference H3K27ac peaks from embryonic and adult tissues (GSE83072, GSE83010, GSE82890, GSE82891, GSE82726, GSE82423, GSE82525, GSE82574, GSE82884, GSE82850, GSM100108, GSM100093, GSM100140, GSM100097). Peaks were converted to $\mathrm{mm} 10$ with liftover. Peaks covering the same genomic region were defined as colocalized using StrandNGS (StrandLifeScience v2.9).

The number of $\mathrm{H} 3 \mathrm{~K} 27 \mathrm{ac}$ peaks outside promoter regions (TSS $\pm 2.5 \mathrm{~kb}$ ) within a $\mathrm{TAD}^{(37)}$ was calculated for $\mathrm{PC}$ and $\mathrm{HC}$. The ratio $(\mathrm{PC} / \mathrm{HC})$ of peaks was used to rank the TADs based on the differential H3K27ac peak number. Information of TADs from ESCs was obtained from GEO (GSE35156 $\left.{ }^{(38)}\right)$. Pairs of H3K27ac peaks were incrementally clustered together if their distance was lower than $12.5 \mathrm{~kb}$. Clusters with at least five H3K27ac peaks were 
annotated as SE. ${ }^{(39,40)}$ Enrichment analysis of enhancer states and SEs was performed using GREAT (http://great.stanford.edu/ public/html, version 3.0.0 $0^{(41)}$ ). Enhancers were assigned to the nearest gene within $1 \mathrm{Mb}$. Enrichment is given as -log 10 of the binomial probability parameters.

To quantify the effect of SEs on differential gene expression, the $B$ of the nearest gene was fitted to a linear model (brms [2.10.0] with default priors ${ }^{(36)}$ and rstan [2.19.2]) (Stan Development Team. 2018. RStan: the R interface to Stan. R package version 2.17.3. http://mc-stan.org).

$$
\begin{gathered}
\beta_{\mathrm{g}} \sim \mathrm{T}\left(\nu, \mu_{\mathrm{g}}, \sigma_{\mathrm{g}}\right) \\
\mu_{\mathrm{g}}=\alpha_{0}+\alpha_{1} * \mathrm{Enh}_{\mathrm{g}} \\
\sigma_{\mathrm{g}}=\gamma_{0}+\gamma_{1} * \mathrm{Enh}_{\mathrm{g}}
\end{gathered}
$$

It models the differential gene expression $\beta$ for a given gene $g$, by a Student's $t$ distribution, defined by $\nu, \mu$, and $\sigma$ (degrees of freedom, mean, and standard deviation, respectively). The slope $\alpha_{1}$ can be interpreted as the effect the presence of a SE (Enh $\left.=1\right)$ has on the differential gene expression compared with its absence $(E n h=0)$. The accuracy of the model was tested by a posterior predictive check. The code is available at https:// github.com/keksundso/SEGeneExpression.

Class I and class II Sox9 peaks (GSE69109 ${ }^{(17)}$ ) within the same genomic region of enhancer states or SEs were defined as colocalization using StrandNGS (StrandLifeScience, version 2.9). To analyze the enrichment of Sox9 peaks in SEs, the overlap (Y) was compared with that of random shuffled genomic sequences of the same length (bedtools $2.26 .0^{(42)}$ ). As an additional control, the Sox 9 peaks were shuffled and the process was repeated. With these data, the following binomial regression model was fitted in a Bayesian framework (brms [2.10.0] with default priors ${ }^{(36)}$ and rstan [2.19.2]) (Stan Development Team. 2018. RStan: the $R$ interface to Stan. $R$ package version 2.17.3. http://mc-stan.org).

$$
\begin{gathered}
\mathrm{Y}_{\mathrm{t}, \mathrm{s}} \sim \operatorname{\operatorname {binomial}}\left(\theta_{\mathrm{t}, \mathrm{s}}, \mathrm{N}_{\mathrm{t}}\right) \\
\theta_{\mathrm{t}, \mathrm{s}}=\operatorname{logistic}\left(\alpha_{\mathrm{t}}+\beta_{\mathrm{t}} * \mathrm{~S}_{\mathrm{t}, \mathrm{s}}\right)
\end{gathered}
$$

$\theta_{t, s}$ is the probability for each type $t$ (PC and HC, each original Sox9 peaks and shuffled peaks) that a SE $(s=1)$ or SE-sized peak $(s=0)$ has a matching Sox9 peak. $N$ is the number of SE peaks and the slope $\beta$ is the log odds ratio of SE peaks $(S=1)$ overlapping with Sox9 compared with randomly located genomic regions $(S=0)$ overlapping with Sox9. The accuracy of the model was tested by a posterior predictive check. The code is available at https://github.com/keksundso/SE_Sox9.

\section{Transcriptome analysis}

Chondrocytes were morphologically distinguished in $10 \mu \mathrm{m}$ cryosections of E14.5 (PC) and E15.5 (HC) embryonic forelimbs and isolated by laser-microdissection with the PALM Microbeam System (Zeiss Microlmaging, Jena, Germany). RNA from fixed chondrocytes sorted by FACS was isolated with the RNeasy FFPE Kit (Qiagen, Hilden, Germany) but yielded low-quality RNA (RIN < 3.2), while laser-microdissection yielded high-quality RNA. RNA was prepared with the RNeasy Micro Kit (Qiagen). RNA integrity was measured with the RNA 600 Pico Kit on a Bioanalyser (Agilent, Waldbronn, Germany). RNA was isolated in several rounds of microdissection from 12 to 16 embryos for each replicate of $\mathrm{PC}$ and 24 to 33 embryos for $\mathrm{HC}$. The mean RINs for the PC replicates were 7.2 and 7.4, ranging from 6.9 to 7.6 per isolation. For $\mathrm{HC}$, the mean RINs were 7.6 and 7.8 , ranging from 7.0 to 8.7. A total of $10 \mathrm{ng}$ of pooled RNA was used for each library preparation with the NuGen Ovation FFPE library system kit and mouse InDA-C primers. Sequencing (RNA-seq) was performed on an llumina HiSeq2500 with 101 cycles (PE mode). The quality of the fastq data was examined with FastQC. Trimmomatic- $0.36^{(43)}$ was used to remove adapters and reads with a quality score below 20 . The reads were then pseudoaligned to $\mathrm{mm} 10$ and quantified using Kallisto, version 0.43.1. ${ }^{(44)}$ Expression levels were calculated as transcripts per million (TPM). Transcripts with an expression value below 1 TPM were removed. Differential expression between PC and HC was analyzed with Sleuth ${ }^{(45)}$ using the Wald test. Significance was defined as $q$ value $<0.05$, with Benjamini-Hochberg-adjusted false discovery rate to correct for multiple comparisons. The code is available at https:/github.com/ yingstat/ScriptsforPaperChondrocytes. The sequencing data are available at the GEO database (GSE155401).

GO enrichment analyses were carried out and visualized using clusterProfiler. ${ }^{(46)}$ Significant enrichment was determined based on $q$ values. For qPCR, cDNA was synthesized with the Maxima First Strand cDNA Synthesis Kit (Thermo Fisher Scientific) using $1 \mathrm{ng}$ RNA. qPCR was performed on the CFX384Touch Real-Time PCR detection system (Bio-Rad, München, Germany) with the my-Budget 5x EvaGreen QPCR-Mix II (Bio-Budget, Krefeld, Germany) using primers listed in Table 1. Expression data were normalized to Beta-2 microglobulin (B2M), and differential expression of four biological replicates was calculated as $-2^{\Delta C T}$. Graphs were plotted with GraphPad (La Jolla, CA, USA) Prism (version 8.4.1). Statistical analysis tested distribution using Shapiro-Wilk test and unpaired, two-tailed Student's $t$ test for significance. Error bars mark the standard deviation between replicates.

\section{In situ hybridization and immunofluorescence staining}

For in situ hybridization, the DIk1 coding sequence was cloned in the pCR4 vector using the TOPO-TA cloning kit (Thermo Fischer Scientific) using the primers D/k1fw: CCTGGCTGTGTCAATGGAGT, DIk1rev: GGTGAGGAGAGGGGTACTC (Metabion, Planegg, Germany). RNA antisense probes were labeled with Digoxigenin11-UTP (Roche, Mannheim, Germany). Hybridization was performed as described previously. ${ }^{(47)}$ Immunofluorescence

Table 1. Primers Used for $\mathrm{qPCR}$

\begin{tabular}{lcc}
\hline Gene name & Fw primer & Rev primer \\
\hline B2M qPCR & GCTCGGTGACC & GAGGCGGGTGGAA \\
& CTGGTCTI & CTGTGT \\
CD44 qPCR & ACTCAGACTCAGG & TCCGTACCAGGCAT \\
& AGCCCAC & CTTCGT \\
Col2a1 qPCR & CGAGTGGAAGAG & AACTICATGGCGT \\
& CGGAGACT & CCAAGGT \\
Col10a1 qPCR & ACGGCACGCCTA & CCATGATTGCACTC \\
& CGATGT & CCTGAA \\
Dlk1 qPCR & TGCGCCAACAATG & GCGGCTACGATCTC \\
& GAACTTG & ACAGAA \\
Sox9 qPCR & AGTACCCGCATCT & TACTGTAATCGGGG \\
& GCACA AC & TGGTCT \\
\hline
\end{tabular}


staining was performed with a rat-CD44 antibody (Invitrogen, Carlsbad, CA, USA) as described previously. ${ }^{(48)}$ Fluorescence pictures were taken on a Zeiss Axio Observer7 microscope with an AxioCam 506 mono CCD camera and Zen2.3 software.

\section{Chondrocyte micromass cultures}

Primary chondrocytes of E12.5 mouse embryos were digested with $1 \mathrm{U} / \mathrm{mL}$ neutral protease NB (Serva) for 15 minutes and with $0.3 \mathrm{U} / \mathrm{mL}$ collagenase NB 4 (Serva) $/ 0.05 \%$ trypsin (Life Technologies, Carlsbad, CA, USA) for 30 minutes at $37^{\circ} \mathrm{C}$. Single-cell suspensions were obtained by passing through a $40-\mu \mathrm{m}$ cell strainer. A total of $2 \times 10^{7}$ cells were cultured in high density as described previously. ${ }^{(49)}$ RNA isolation and Alcian blue (Merck, Darmstadt, Germany) staining ${ }^{(50)}$ was performed after 4 and 7 days of differentiation. Cells were treated with $120 \mu \mathrm{M} \mathrm{CD44}$ inhibitor Verbascoside (Taufkirchen, Germany) ${ }^{(51)}$ during differentiation. Images were taken on a Zeiss Axioplan 2 microscope with a SPOT 14.2 camera and SPOT advanced software (Diagnostic Instruments, Sterling Heights, MI, USA). Alcian blue was extracted with $6 \mathrm{M}$ guanidine hydrochloride (Merck) and measured on a GeniosPro reader (Tecan, Mannedorf, Switzerland) in technical duplicates. qPCR was performed as described above in four biological replicates. Results are given as foldchange over control. Unpaired, two-tailed Student's $t$ test was used to calculate significance. Error bars mark standard deviation between replicates.

\section{Correlation of gene expression and H3K36me3 occupancy}

To analyze the effect the H3K36me3 load and the distance between the H3K36me3 and the start of a given gene (g) have on its expression (mload and mdist, respectively), we fitted a linear model in a Bayesian framework using rstan (2.19.2) (Stan Development Team. 2018. RStan: the R interface to Stan. R package version 2.17.3. http://mc-stan.org). The distance was defined as the scaled logarithm of the number of base pairs between the gene start (retrieved with biomaRt [version 2.42.1] ${ }^{(52,53)}$ dependent on strandness) and the closest called H3K36me3 peak. The load was defined as the scaled logarithm of the number of H3K36me3 reads on the gene body (normalized by library size). We chose weakly informative priors with a normal $(0.0,10.0)$ for the intercept (alpha), mdist and mload, exponential(0.5) for the standard deviation (sigma), and gamma(2, 0.1) for the degrees of freedom ( $\nu$ ).

$$
\begin{aligned}
& \operatorname{scaled}\left(\log \left(\operatorname{tpm}_{\mathrm{g}}\right)\right) \sim \mathrm{T}\left(\nu, \mu_{\mathrm{g}}, \sigma\right) \\
& \mu_{\mathrm{g}}=\text { alpha } \text { mdist*dist }_{\mathrm{g}}+\text { mload*load }_{\mathrm{g}}
\end{aligned}
$$

\section{Correlation between gene expression and ChromHMM state prevalence}

The genomic regions (gene: transcribed region, ENSEMBL), TSS $( \pm 2.5 \mathrm{~kb})$ and enhancer (transcribed region $\pm 10 \mathrm{~kb}$ ) of a given gene were described by a binary vector with 15 elements, with each element of the vector representing the prevalence of a particular ChromHMM state, with an element set to 1 if the region overlaps a bin of that ChromHMM state and 0 otherwise. To associate ChromHMM state prevalence with gene expression levels, the TPM values of each gene were sorted by increasing TPM values and ranked accordingly. The TPM ranks were split into non-overlapping sets of 500 ranks (genes). For each gene set, the mean prevalence of the different ChromHMM states and the mean TPM value was computed using bootstrapping ( $R$ package boot version 1.3-23, number of bootstrap replicates $R=10,000$ ). The $95 \%$ highest-density intervals (HDIs) of the mean state prevalence are given.

\section{Integrative analysis of ChIP-seq and RNA-seq data}

The integrative analysis was done with $\mathrm{R}$ package intePareto (https://github.com/yingstat/intePareto). ${ }^{54}$ Counts were estimated from the raw RNA-seq data (FASTQ format) with Kallisto (version $0.43^{(44)}$ ). The raw ChIP-seq data in FASTQ format were aligned to $\mathrm{mm} 10$ with BWA $\left(0.7 .15^{(26)}\right)$ and sorted with Samtools (version 1.5 $\left.{ }^{(55)}\right)$. To define the epigenetic signal for the number of ChIP-seq reads of each mark falling into the promoter region (TSS $\pm 2.5 \mathrm{~kb}$ ) were calculated. Matching of RNA-seq and ChIPseq data on the gene level was performed with "highest" strategy implemented in intePareto. The fold-change was calculated for each histone mark with DESeq2 (version 1.24.0 ${ }^{(56)}$ ), and assigned a $Z$-score, reflecting the differential coverage between $\mathrm{PC}$ and $\mathrm{HC}$ in relation to the fold-change in RNA-seq. Genes were ranked by Pareto front in the space defined by all $Z$-scores. The code is available at https://github.com/yingstat/ ScriptsforPaperChondrocytes.

\section{Results}

Cell type-specific epigenetic profiles of chondrocytes

To identify epigenetic changes linked to the differentiation of PC into $\mathrm{HC}$, the axial and appendicular skeletons of mice expressing YFP under the control of the Col2a1 (Col2-Cre;R26R-YFP) or the Col10a1 (Col10-Cre;R26R-YFP) promoter were dissected. Flow cytometry of cells isolated from Col2-Cre;R26R-YFP embryos at E13.5, a time point before hypertrophic differentiation takes place, detected about $15 \%$ to $20 \%$ YFP-positive PC. These were enriched to about $90 \%$ by FACS. HC were isolated from E15.5 Col10-Cre;R26R-YFP embryos before the transdifferentiation into osteoblasts takes place. In accordance with the low fraction of $\mathrm{HC}$ in E15.5 skeletons, only $1 \%$ of the isolated cells were YFP positive. These were enriched to approximately $80 \%$ YFP-positive cells (Fig. $1 A, B$ ). Enrichment of the respective cell population was verified by qPCR of Sox 9 and Col2a 1 for PC and Col10a 1 for HC (Supplemental Fig. S1A-C).

For epigenetic profiling, we investigated the distribution of the activating promoter marks $\mathrm{H} 3 \mathrm{~K} 4 \mathrm{me} 3$ and $\mathrm{H} 3 \mathrm{~K} 9 \mathrm{ac}$, the promoter and enhancer mark H3K27ac, the transcription-associated mark $\mathrm{H} 3 \mathrm{~K} 36 \mathrm{me} 3$, and the repressive marks $\mathrm{H} 3 \mathrm{~K} 9 \mathrm{me} 3$ and H3K27me3 by ChIP-seq (Supplemental Fig. S1D). Due to the restricted number of $\mathrm{HC}$ per embryo, the ChIP-seq experiments were performed in biological triplicates for $\mathrm{PC}$ and in duplicates for HC. Principal component analysis and hierarchical clustering revealed clustering according to distinct histone marks (Supplemental Fig. S1E, F) and a high similarity between the activating and repressive modifications, respectively. H3K36me3 showed a similar correlation with both groups (Supplemental Fig. S1F), likely reflecting the potential overlap with activating and repressive marks. ${ }^{(16)}$

Genomic regions enriched for each chromatin mark (Supplemental Table S1) were identified by hiddenDomains. ${ }^{(32)}$ Peak distribution analysis detected the expected enrichment of activating promoter marks and the repressive modification 

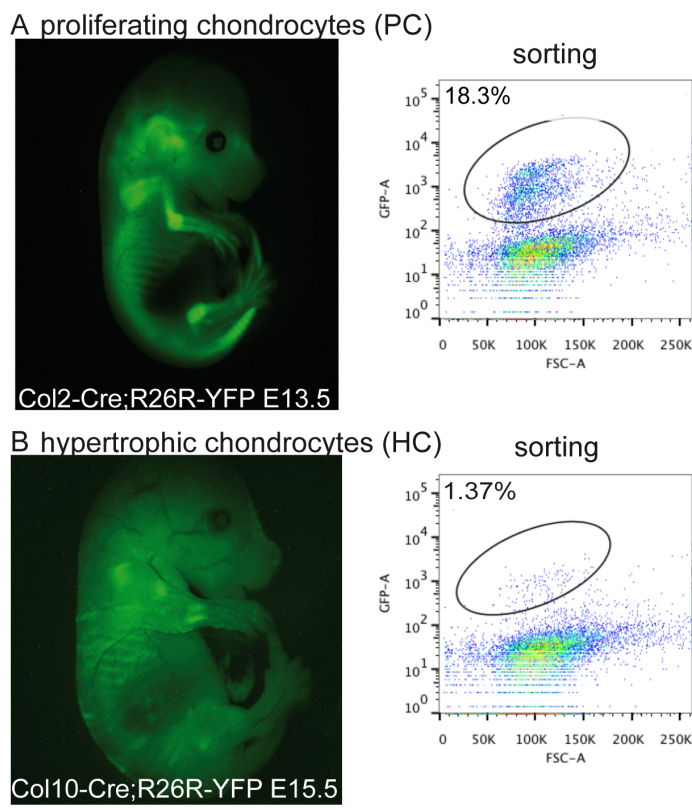

D
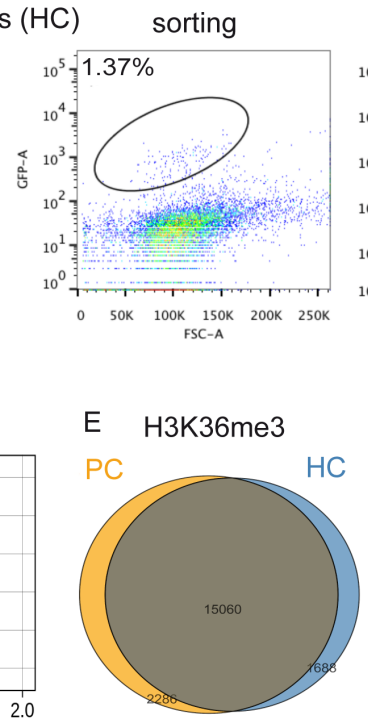

$\begin{array}{ccccc}\text { - triplicate } & 0.0 & 0.5 & 1.0 & 1.5 \\ \text { ratio } & (\mathrm{PC} / \mathrm{HC})\end{array}$

G

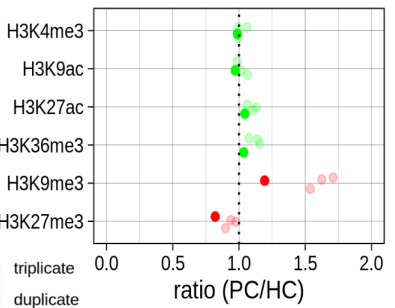

$$
\text { PC }
$$

H3K4me3

H3K9ac

H3K27ac

H3K36me3

H3K9me3

H3K27me3

Sox9 chr11:112.779.232-112.789.194

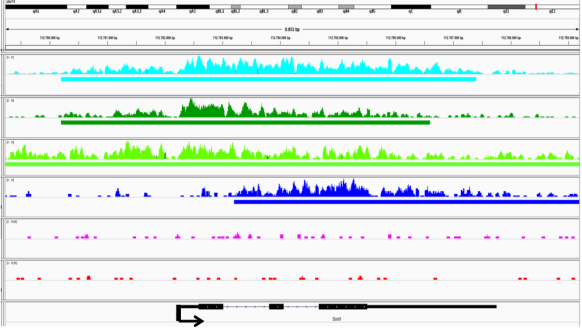

$\mathrm{H}$

$H$

H3K4me3

H3K9ac

H3K27ac

H3K36me3

H3K9me3

H3K27me3
Sox5 chr6: $144.162 .690-144.211 .910$

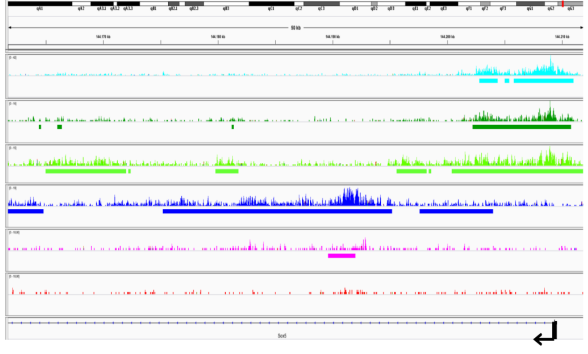

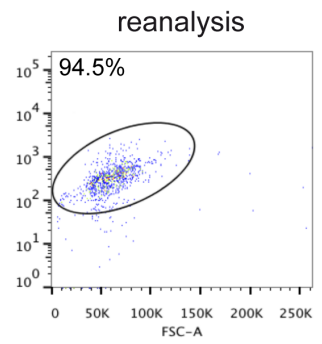

C

Figure 1

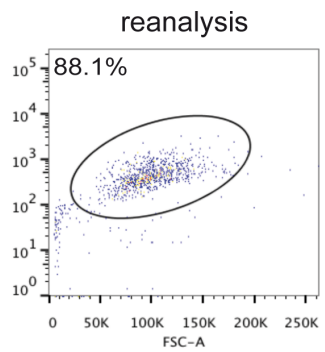

$\mathrm{F}$ all activating marks

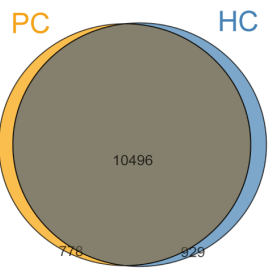

Cell type $\square$ PC $\square$ HC
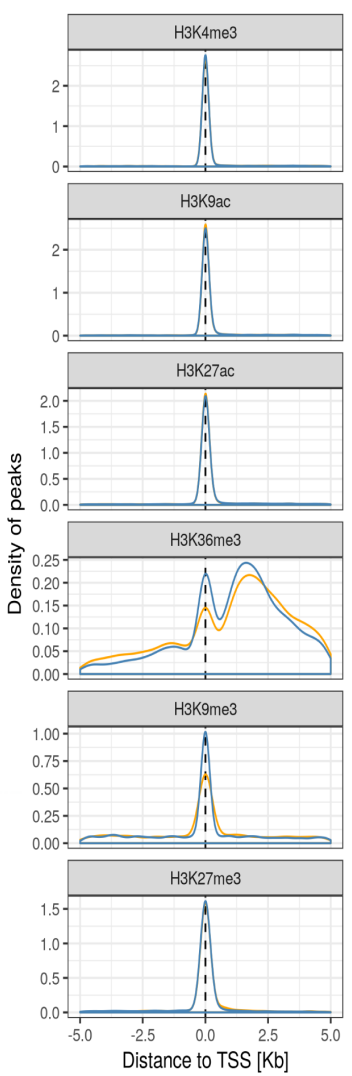
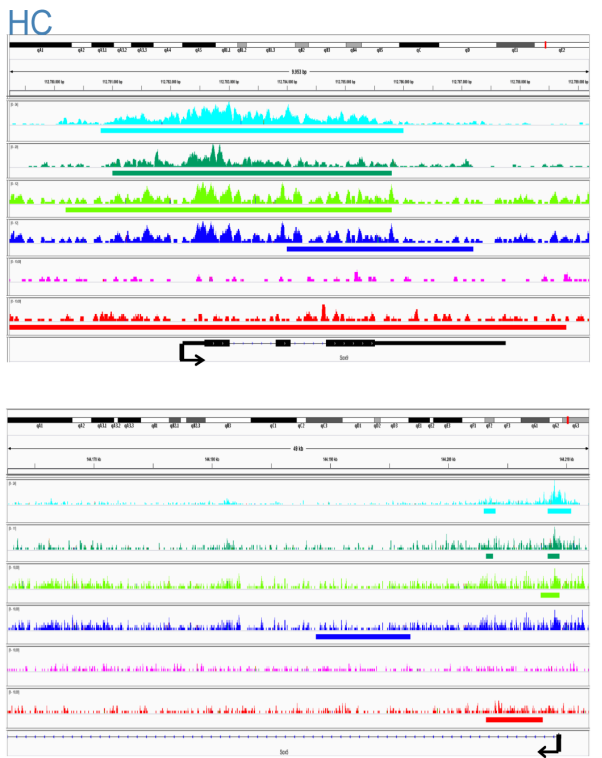

Fig 1. Epigenetic profiling of $P C$ and HC. $(A, B)$ Fluorescent images and flow cytometry of cells isolated from E13.5 Col2-Cre;R26R-YFP $(A)$ and E15.5 Col10-Cre;R26RYFP (B) mice before (sorting) and after cell sorting (reanalysis). Black ellipse: YFP-positive populations. (C) Peak density of histone marks around the TSS ( $\pm 5 \mathrm{~kb}$ ) in PC (orange) and $\mathrm{HC}$ (blue). ( $(D)$ Ratio (PC/HC) of genes marked by the indicated histone modification. Green: activating marks; red: repressive marks. ( $E, F)$ Venn diagram of genes carrying H3K36me3 ( $E$ ) or the activating histone marks H3K4me3, H3K9ac, H3K27ac, and H3K36me3 ( $F$ ) in PC (orange) and HC (blue). ( $G$, $H$ ) Read and peak coverages of histone marks in the genomic regions of Sox9 $(G)$ and Sox5 $(H)$ visualized in IGV. Black arrow: TSS. 
$\mathrm{H} 3 \mathrm{~K} 27 \mathrm{me} 3$ at the transcription start site (TSS $\pm 1 \mathrm{~kb}$ ), whereas $\mathrm{H} 3 \mathrm{~K} 36 \mathrm{me} 3$ and H3K9me3 were distributed throughout the genome (Fig. 1C). To exclude that different replicate numbers in $\mathrm{PC}$ and $\mathrm{HC}$ affect the analysis, we compared peak coverage and associated gene number of the PC data sets pairwise but did not detect major differences between the PC triplicate and the different subsets of PC duplicates (Fig. 1D; Supplemental Fig. $S 2 A, B)$. We compiled a conserved data set of peaks that were detected in the respective replicates and used this data set for further analyses. Comparison of peak coverage and gene number revealed a similar distribution of most modifications in PC and $\mathrm{HC}$ and a slightly increased number of $\mathrm{H} 3 \mathrm{~K} 27 \mathrm{me} 3$ peaks in HC (Fig. 1D; Supplemental Fig. S2A-E).

Analysis of genes marked as transcribed by $\mathrm{H} 3 \mathrm{~K} 36 \mathrm{me} 3$ identified 2286 genes specifically expressed in PC and 1688 in HC, whereas 15,060 genes were expressed in both cell types, reflecting the common chondrogenic lineage (Fig. 1E). A large fraction of these genes was additionally marked by the three activating promoter marks (Fig. 1F). Evaluation of genes known to be downregulated in $\mathrm{HC}$, like Sox9 and Sox $5^{(17,50)}$ revealed a high coverage of activating promoter marks at the TSS and H3K36me3 on the gene body in $\mathrm{PC}$, whereas repressive marks were absent. Interestingly, in $\mathrm{HC}$, these genes gain the repressive mark H3K27me3 especially at the promoter region but remain decorated with reduced levels of activating marks (Fig. $1 G, H$ ), indicating that gene repression is initiated by the addition of $\mathrm{H} 3 \mathrm{~K} 27 \mathrm{me} 3$ in regions still marked as active. In contrast, genes not expressed in chondrocytes, like NeuroD4, did not carry repressive marks in either cell type (Supplemental Fig. S6C), making an unspecific increase in histone methylation during differentiation unlikely.

\section{Genes marked as active gain H3K27me3 during differentiation}

To identify epigenetic changes linked to differentiation in an unbiased approach, we defined 15 chromatin states carrying distinct combinations of epigenetic marks using ChromHMM (Fig. 2A; Supplemental Fig. S3A, $B$; Supplemental Fig. S4A-C). Based on the combination of histone marks, the chromatin states were classified as activating (states $8,9,11,13$ ), enhancer (states $5,7,10$ ), expressed (states 3,4$)$, repressive (states 1, 2, 14), and empty (state 6). In addition, ChromHMM identified two chromatin states with a combination of $\mathrm{H} 3 \mathrm{~K} 27 \mathrm{me} 3$ and activating promoter marks (state 13) or H3K36me3 (state 15), here referred to as repressed-active states (Fig. $2 A$ ). In the same cell type, transitions between states along the genome occurred with highest probability between functionally related states or into the empty state 6 (Supplemental Fig. S3C). Functional assignment revealed the expected, region-specific enrichment of promoterassociated states 8 to 13 at the TSS and CpG islands. The enhancer states 5, 7, and 10 colocalized with published enhancers extracted from the Vista and Fantom databases (Supplemental Fig. S3D). To confirm that the identified ChromHMM states represent histone combinations frequently occurring during development, we included epigenetic data from E11.5 to E15.5 limb bud and brain tissue into the model. Besides the detection of more promoter states, we did not find major differences in the generalized model. The most interesting difference was the loss of the repressed-active state 15, indicating that the combination of activating and repressive histone marks was overrepresented in our data set (Supplemental Fig. S4D).
We next asked if hypertrophic differentiation is associated with distinct changes in the epigenetic profile. Comparing the histone states of the two cell populations revealed more states defined by activating marks in PC, whereas repressive and repressed-active states were more frequent in $\mathrm{HC}$ (Fig. $2 A, B$ ). This is in good agreement with the observation that genes downregulated in $\mathrm{HC}$ gain $\mathrm{H} 3 \mathrm{~K} 27$ me3 while maintaining activating histone marks (Fig. 1G, H). We tested if this gain in repressive states is characteristic for chondrocyte-specific genes by calculating the enrichment of ChromHMM state transitions between $\mathrm{PC}$ and $\mathrm{HC}$ in a set of 334 chondrogenesis-associated genes and compared it to the enrichment in a set of housekeeping genes $^{(35)}$ (Supplemental Table S2). For the housekeeping genes, transitions between the activating states 4, 9, and 11 showed the highest enrichment (Fig. 2C; Supplemental Fig. S4E). Likewise, transitions between activating $(5.4,4.5)$, activating and empty $(4.6,6.4,5.6,7.6)$, and the same $(1.1,4.4,5.5,9.9$, and 13.13) states were enriched in the set of chondrocyte-specific genes, likely reflecting the similar expression pattern in the closely related cell types (Fig. 2D; Supplemental Fig. S4F). Transitions between the silencing state 1 and the repressed-active promoter state 13 also occurred rather frequently in both directions, pointing to a high variability of marks on permanently repressed genes (Supplemental Table S3).

Importantly, transitions from the activating promoter states 9 and 11 to the repressed-active promoter state 13 were unidirectionally enriched in the chondrocyte-specific data set (Fig. 2D). Genes undergoing this transition included several genes known to be downregulated in $\mathrm{HC}$ like Sox5, Sox6, Sox9, and Ptch1 (Supplemental Table S3). Enrichment analysis revealed an association with non-canonical Wnt-signaling and Myc/Maz transcription factors, which have been linked to the switch from PC to HC (Fig. 2E). ${ }^{(57,58)}$ In summary, the gain of H3K27me3 on promoters still decorated with activating marks seems to be specifically linked to the repression of transcription.

\section{The gain of H3K27me3 initiates gene repression}

To correlate changes in epigenetic states and gene expression, we analyzed the transcriptome of $\mathrm{PC}$ and $\mathrm{HC}$ isolated by laser microdissection from E14.5 and E15.5 mouse forelimbs by RNAseq in duplicates for each cell type (Fig. $3 A, B$ ). Similar read numbers were obtained for all data sets and a high correlation was detected between replicates (Supplemental Fig. S5A, B). Many genes known to be differentially expressed, like Sox5, Wwp2, and Matn4 in PC and Col10a1, Mef2c, Foxp1, and Mmp9 in HC were found in the respective data set (Fig. 3C). Furthermore, differentially expressed genes were highly enriched in GO terms related to bone development and ossification reflecting the chondrogenic cell type (Fig. 3D). To identify new potential regulators of chondrogenesis, we inspected the topmost 30 differentially expressed genes in detail. Of these, 8 were included in cartilage differentiation associated GO terms and 11 have previously been described in chondrocytes, whereas the remaining genes represent potential novel regulators of chondrocyte differentiation (Supplemental Table S4). Spearman-rank correlation detected a positive correlation between the RNA-seq data and the activating but not the repressive histone marks (Supplemental Fig. S1F). Interestingly, we also found a positive correlation between the differential levels of gene expression and the occupancy of activating promoter and enhancer marks. Furthermore, differential H3K27me3 occupancy negatively correlated with those of the activating marks (Fig. 3E). Genes 


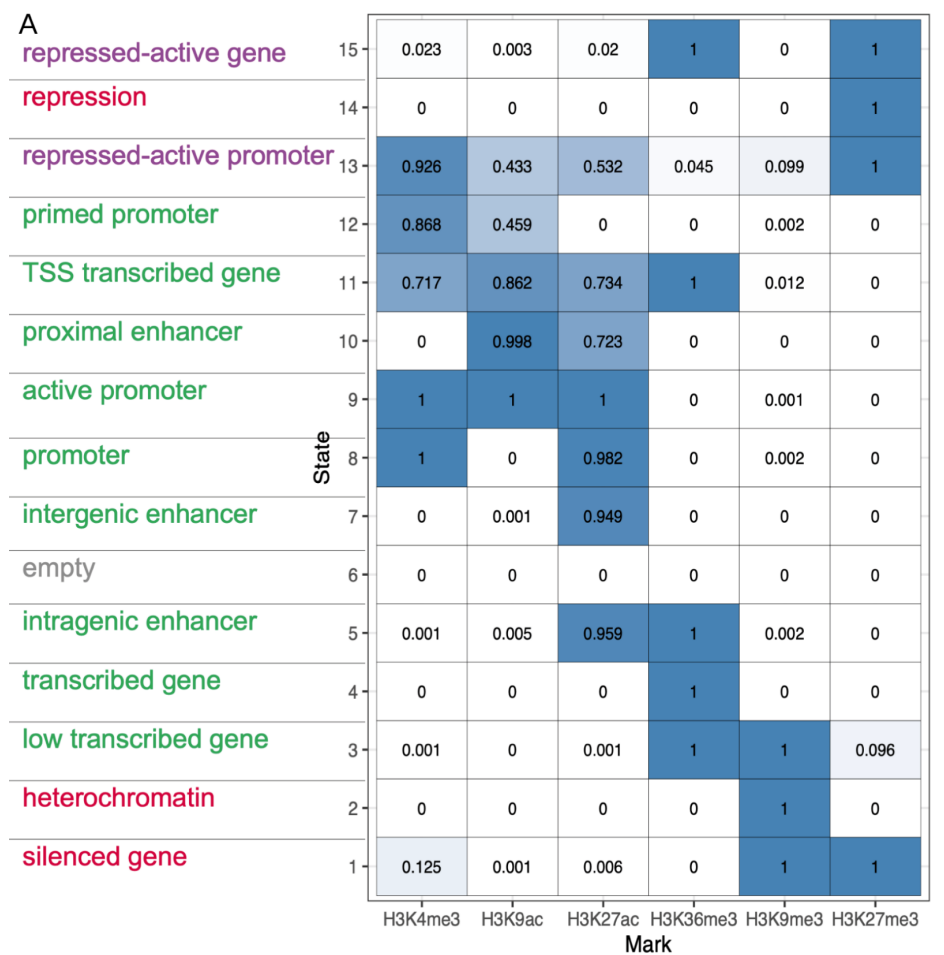
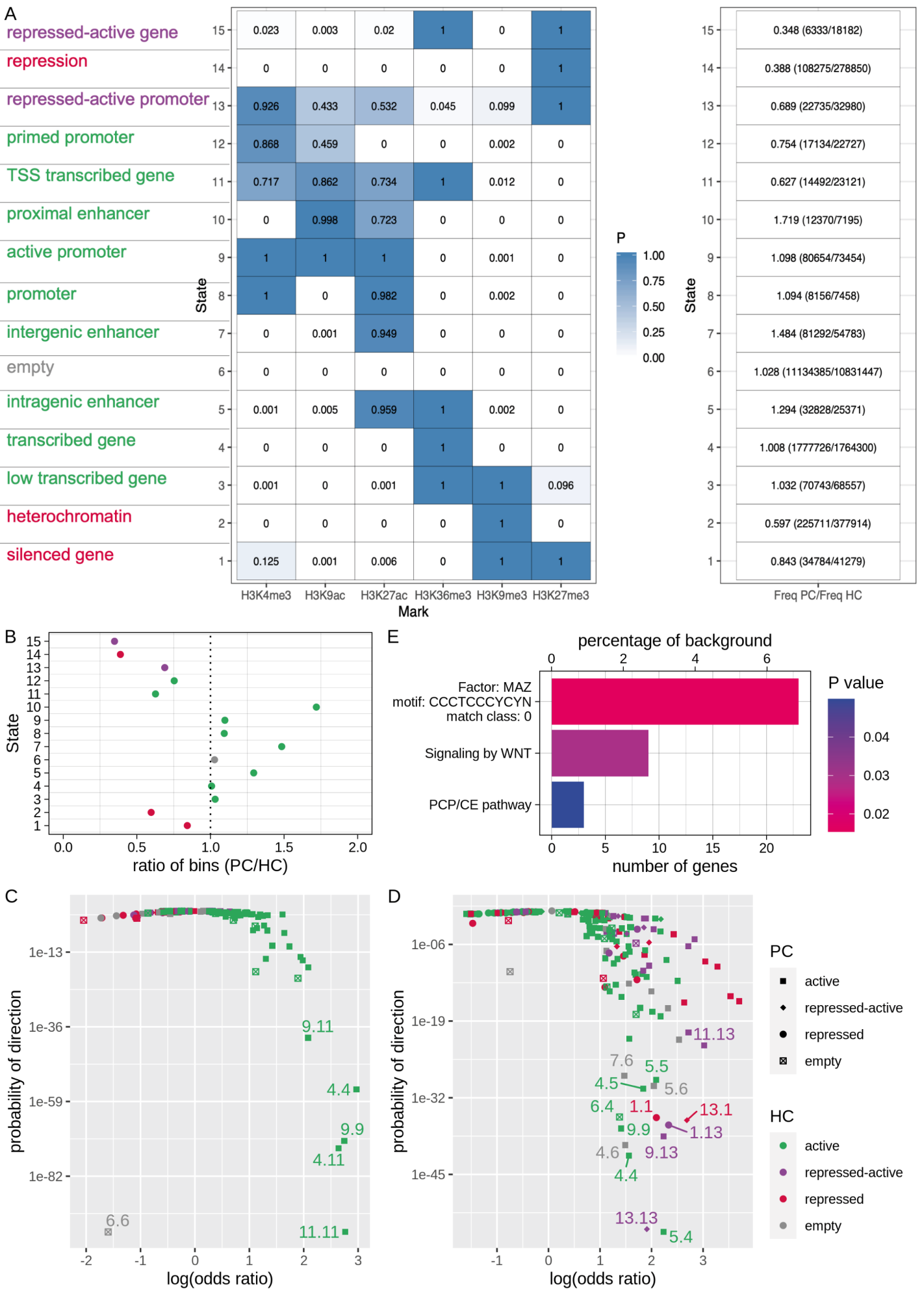

Fig 2. Enrichment of ChromHMM state transitions from active to repressed-active states at chondrocyte-specific genes. (A) Emission probabilities of a 15-state ChromHMM model based on the conserved PC and HC data sets. Left column: functional annotations of states. Green: activating states; red: repressive states; purple: repressed-active states; gray: empty state. Right column: ratio and frequencies of the respective states in PC and HC. (B) Ratio (PC/HC) of genomic bins in each ChromHMM state. $(C, D)$ Enrichment or depletion of each ChromHMM state transition in a given set of 265 housekeeping genes $(C)$ and 334 chondrogenesis-associated genes $(D)$ (listed in Supplemental Table S2). The log(odds ratio) is plotted against the probability of direction (enrichment or depletion). (E) Enrichment analysis of chondrogenic genes undergoing the transition from the activating promoter state 9 and the expressed state 11 in PC to the repressed-active promoter state 13 in $\mathrm{HC}$. 
identified as transcribed by RNA-seq were decorated with H3K36me3 in both cell types (Fig. $3 F-H$ ) and the expression level showed a slight correlation with the quantitative occupancy of H3K36me3 (positive) and the distance of this mark to the start of the gene (negative) (Supplemental Fig. S5C-F). However, the uncertainty in the prediction was very high (Supplemental Fig. S5C, D) and differential H3K36me3 occupancy did not correlate with changes in gene expression (Fig. $3 E$ ). The presence of $\mathrm{H} 3 \mathrm{~K} 36 \mathrm{me} 3$ seems thus to reliably predict gene expression, whereas the degree of $\mathrm{H} 3 \mathrm{~K} 36 \mathrm{me} 3$ occupancy is less informative.

Analyzing the distribution of ChromHMM states, we found a positive correlation between TPM values and the prevalence of promoter, enhancer, and transcription-associated states at the promoter region (TSS $\pm 2.5 \mathrm{~kb}$ ), while repressive histone states were enriched on genes with low TPM values. Similarly, the repressed-active state 13 was prevalent on genes with intermediate to low TPM values (Fig. 3/; Supplemental Fig. S5G). Based on the transition analysis, we hypothesized that the gain of H3K27me3 associated with the transitions 9.13 (active/ repressed-active promoter) and 11.13 (TSS transcribed gene/ repressed-active promoter) (Fig. 2D) lead to the downregulation of PC-specific genes in HC. To confirm this hypothesis, the differential expression of genes undergoing these transitions was compared with their published expression patterns and many of these genes, like Sox 9 and Sox5, showed the expected downregulation in $\mathrm{HC}$, while another set of genes was higher expressed in HC (Fig. 4A; Supplemental Table S3). Many genes in this group, like $P$ th $1 r$, are known markers of prehypertrophic chondrocytes $(\mathrm{PHC})$, which are upregulated with the onset of hypertrophy and repressed at later hypertrophic stages. These data indicate that PHC are present in the HC pool of the transcriptome analysis, wheras they are enriched in the PC population used for epigenetic profiling (Fig. 4B).

ChromHMM states combine histone modifications at specific genomic locations, but the number of reads detected for each modification is not considered. To quantitatively correlate alterations in epigenetic modifications with differential expression, we integrated the differential level of RNA-seq and promoterspecific ChIP-seq reads (TSS $\pm 2.5 \mathrm{~kb}$ ) by Pareto optimization. Because the differential occupancy of H3K36me3 and H3K9me3 did not correlate with differential gene expression (Fig. 3E), these marks were excluded from the analysis. The genes were sorted into Pareto fronts based on their positive (for the activating marks) or negative (for H3K27me3) Z-scores (Supplemental Table S5). Many genes known to be differentially expressed, like Sox5, Sox9, and Col10a1, were found in the topmost-ranking Pareto fronts (Fig. 4C). These fronts also included genes with epigenetic profiles similar to those of Sox5 and Sox9, like Delta-like kinase 1 (Dlk1; Supplemental Fig. $\mathrm{S} 6 A)^{(59,60)}$ in $\mathrm{PC}$ or to Col10a1, like CD44, in $\mathrm{HC}$ (Supplemental Fig. S6B). ${ }^{(51,61,62)}$ The cell type-specific expression and function of DIk1 and CD44 was confirmed by qPCR, in situ expression analyses, antibody staining, and micromass cultures (Fig. 4D-l; Supplemental Fig. S6D-l). Together these results strongly support the hypothesis that repression of chondrocyte-specific genes is initiated by the gain of H3K27me3 on promoters still carrying a combination of at least three activating marks.

Surprisingly, the lowest-ranking Pareto fronts included several genes, like Runx2,Mef2c, Trps1, and Pth1r, which are specifically upregulated in $\mathrm{PHC}$ and are subsequently downregulated in HC (Fig. 4C; Supplemental Table S5). These genes also undergo the transition from an activating to a repressed-active state
(Fig. 4A; Supplemental Table S3). The repressed-active state in $\mathrm{HC}$ corresponds to the downregulation of expression in these cells. Closer inspection of individual $Z$-scores revealed a negative correlation of differential mRNA expression and activating marks further supporting the hypothesis that $\mathrm{PHC}$ were included in different cell pools used for RNA-seq $(\mathrm{HC})$ and epigenetic characterization (PC) (Fig. 4B). While supporting the repressed-active state as an indicator of early gene repression, the different sampling methods led to a specific accumulation of transition associated genes in the lowest-ranking Pareto fronts (Fig. 4C), making them a source of new potential regulators of the switch in cell state.

\section{HC-specific enhancers regulate metabolic pathways}

Based on H3K27ac occupancy, ${ }^{(63)}$ we defined three enhancer states, the proximal enhancer state 10, which included the promoter mark H3K9ac and was mainly found close to the TSS, and the distal enhancer states, state 5 (intragenic enhancer) and state 7 (intergenic enhancer), which were located at distances of up to $>500 \mathrm{~kb}$ outside the next TSS (Fig. 5A, B; Supplemental Fig. S7A). All enhancer states were more prevalent in PC and correlated positively with the expression of the nearest gene $( \pm 1 \mathrm{Mb})$ in both cell types (Supplemental Fig. S7A; Supplemental Fig. S5G). Classifying enhancers as common (22\%), PC-specific (51\%), and HC-specific (27\%) revealed substantial differences in their genomic localization between the cell types (Supplemental Fig. S7A, B). As expected, most proximal enhancers were found close to the TSS. Interestingly, HC-specific, distal enhancer states were located closer to the TSS ( 5 to $50 \mathrm{~kb}$ ) than PC-specific, distal enhancer states, which were more frequently found at larger distances ( $>500 \mathrm{~kb}$ ) (Fig. 5A, B). Enrichment analysis using GREAT assigned proximal enhancer states of both cell types to genes regulating cellular functions like DNA-, RNA-, and proteinmetabolism (Fig. 5C). The common, distal enhancer states were linked to pathways relevant for both cell types, like skeletal and blood vessel development. In contrast, PC-specific, distal enhancer states were associated with genes of chondrogenesis-related pathways, whereas the HC-specific, distal enhancer states regulated metabolic processes, likely required for the increase in cell volume and ECM remodeling in HC (Fig. 5D).

To investigate the conservation of H3K27ac peaks, which include promoter and enhancer regions, we compared the chondrocyte-specific H3K27ac peaks to those of different embryonic and adult tissues. Overall, a high fraction of common H3K27ac peaks, which were enriched at the promoter region (Supplemental Fig. S7C), overlapped with H3K27ac peaks in pre- and postnatal tissues $(62 \%$ to $88 \%$ in embryonic, $52 \%$ to $63 \%$ in adult tissue), while tissue-specific enhancers showed less conservation, especially in postnatal tissues (Fig. 5E). Comparing the relative distribution of cell type-specific enhancers revealed that a higher percentage of PC-specific H3K27ac peaks colocalized with peaks in actively proliferating and developing tissues, like limb and brain, whereas more HC-specific H3K27ac peaks were conserved in early differentiating embryonic tissues like heart and liver and in postnatal tissues (Fig. 5E). In combination with the pathway analysis of common and cell type-specific H3K27ac peaks (Supplemental Fig. S7D), these data indicate that tissue maturation is linked to a switch from differentiation regulating pathways to general cellular and metabolic functions.

Super-enhancers (SEs), defined as clusters of H3K27ac peaks, ${ }^{(40)}$ are important regulators of differentiation. We detected 360 SEs specifically in PC, 205 in HC, and 158 shared 


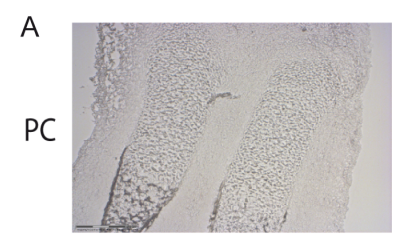

B

$\mathrm{HC}$

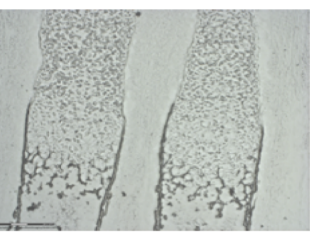

D

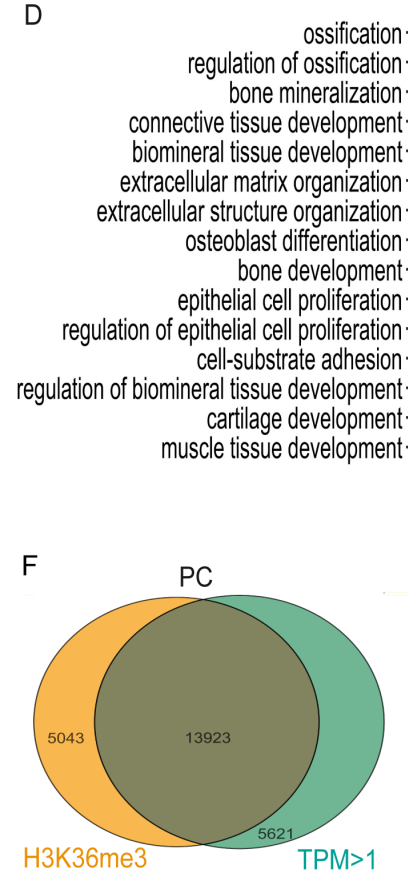

F
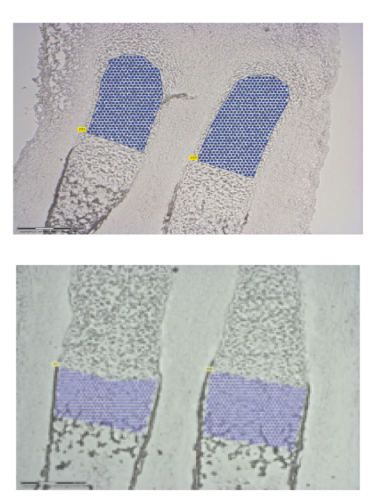

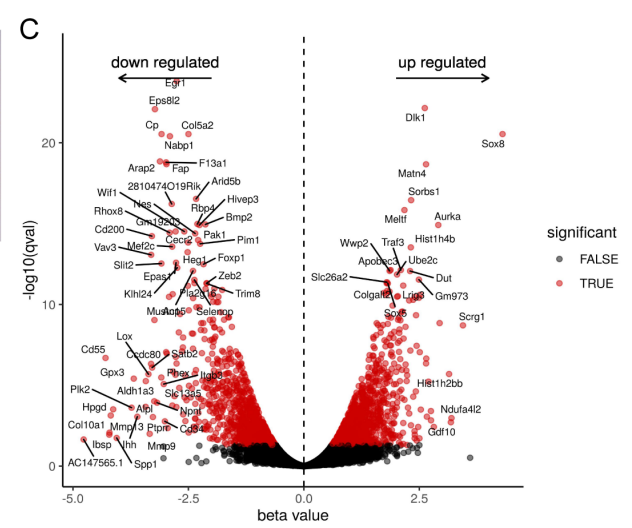

E

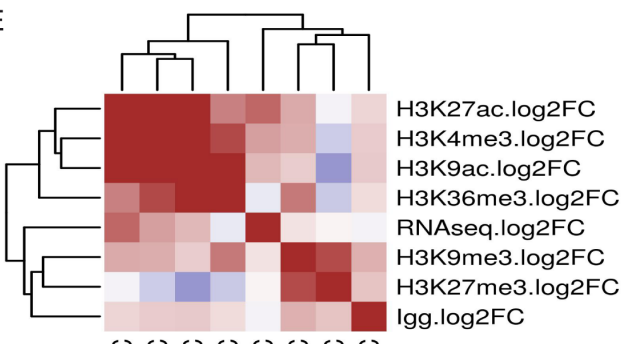

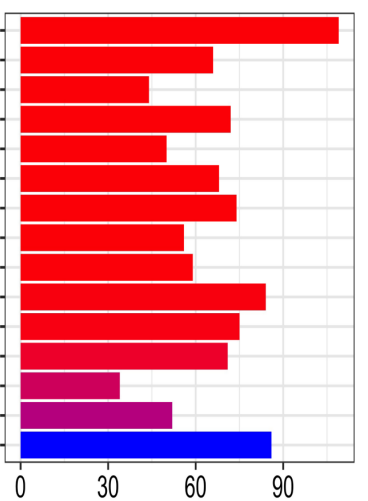

G

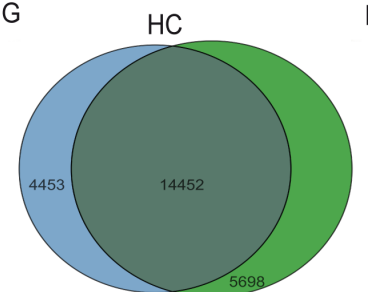

H3K36me3
$\mathrm{TPM}>1$

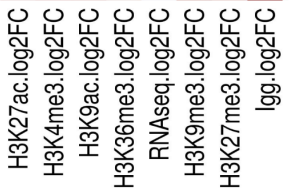

$\mathrm{H}$

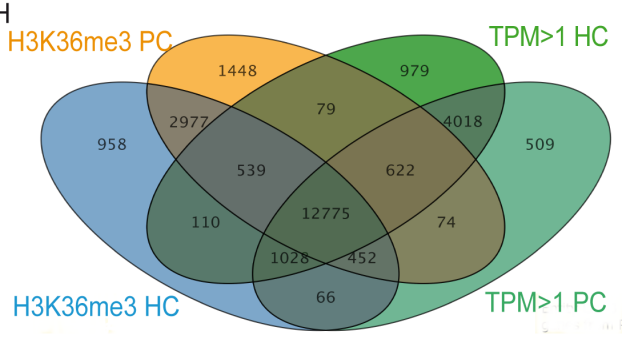

TSS +/- $2.5 \mathrm{~kb}$

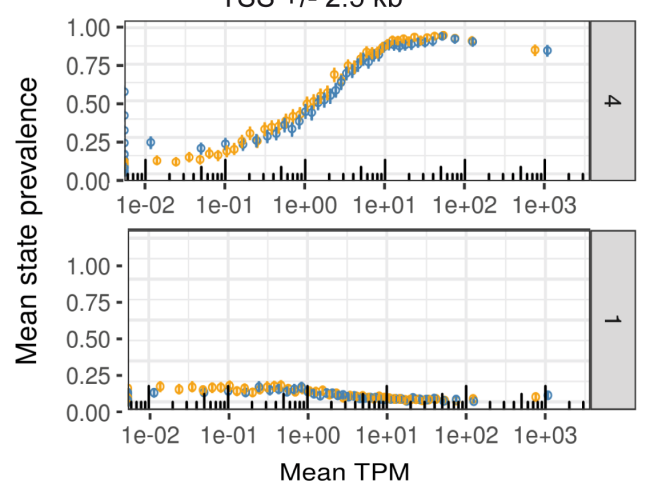

TSS +/- $2.5 \mathrm{~kb}$
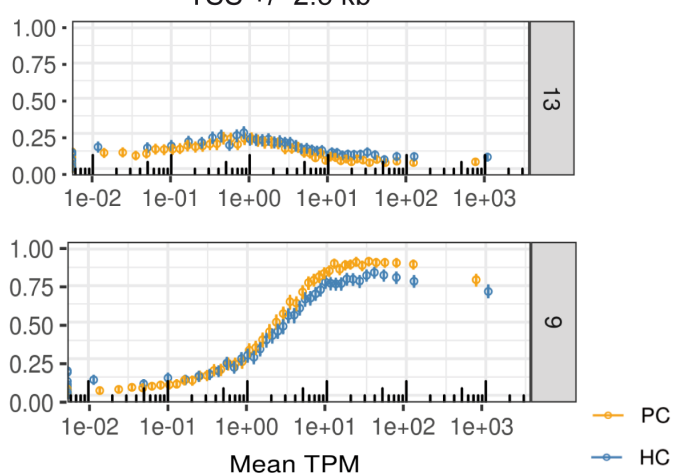

Fig 3. Correlation of differential gene expression and epigenetic modifications. $(A, B)$ Chondrocytes were isolated by laser-microdissection from cryosections of E14.5 (PC, A) or E15.5 (HC, B) forelimbs based on morphology. (C) Volcano plot of differential gene expression between PC (beta $>0$ ) and HC $($ beta $<0$ ). Fifty genes with the highest differences (beta value) and confidence (-log10 $q$ value) are annotated. (D) GO terms with the highest enrichment of genes differentially expressed between PC and HC. (E) Spearman correlation of differential gene expression and differential occupancy of the indicated histone marks. $(F-H)$ Venn diagrams of genes identified as expressed by RNA-seq (green) and genes carrying H3K36me3 in PC (orange; $F$ ) or HC (blue; $G$ ). Comparison of all data sets $(H)$. (I) TPM values of genes assigned to a specific ChromHMM state (silenced: 1; expressed: 4; active promoter: 9; repressedactive promoter: 13 ) at the promoter region (TSS $\pm 2.5 \mathrm{~kb}$ ) in PC (orange) and HC (blue). Mean state prevalence was plotted against mean log(TPM). 

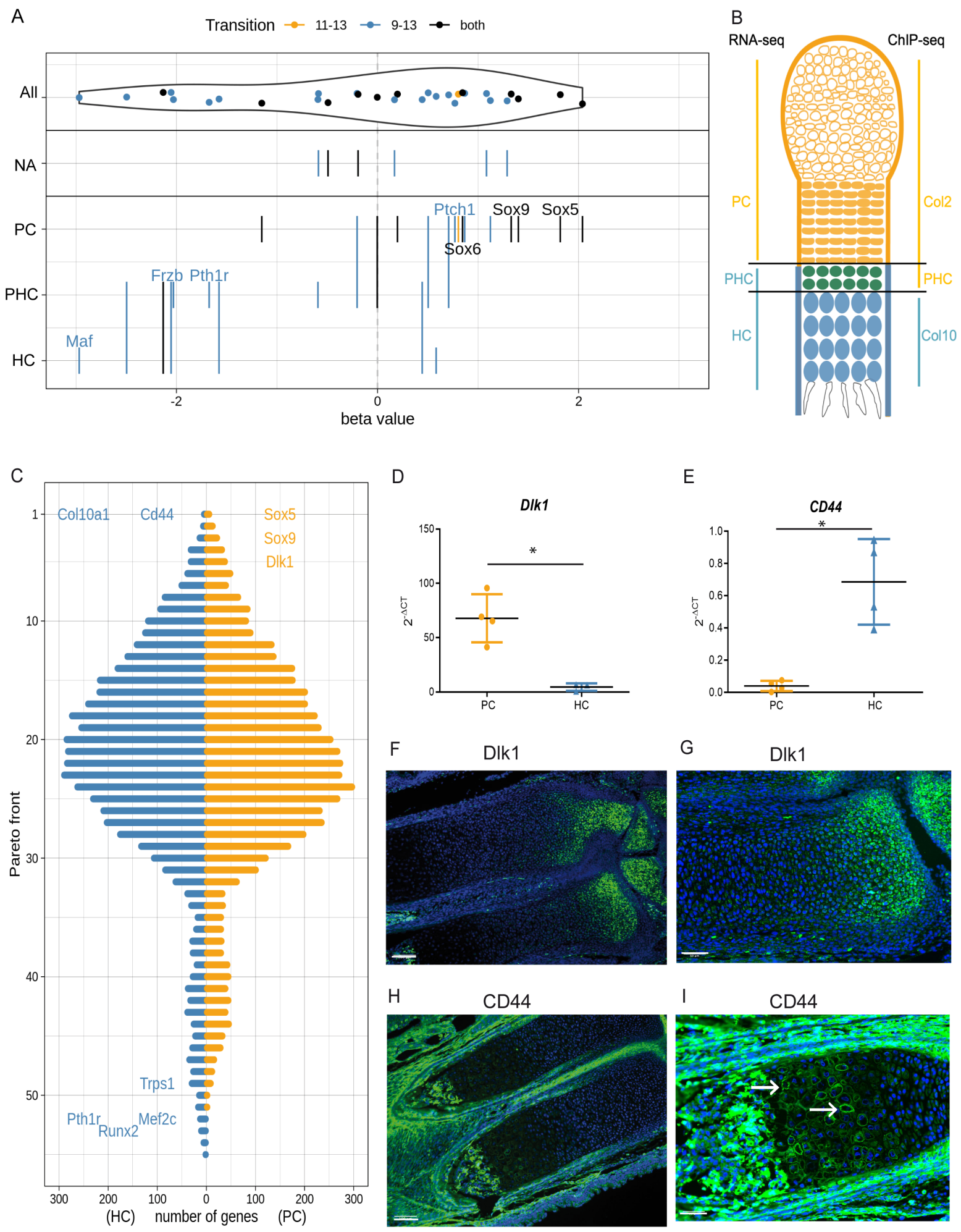

D

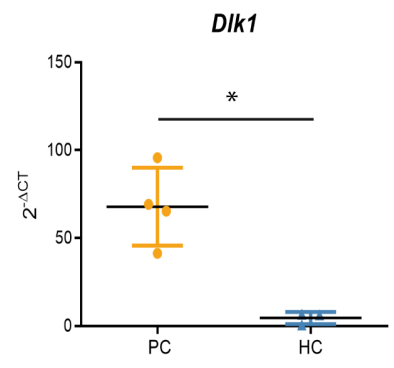

E

$\mathrm{F}$

Dlk1
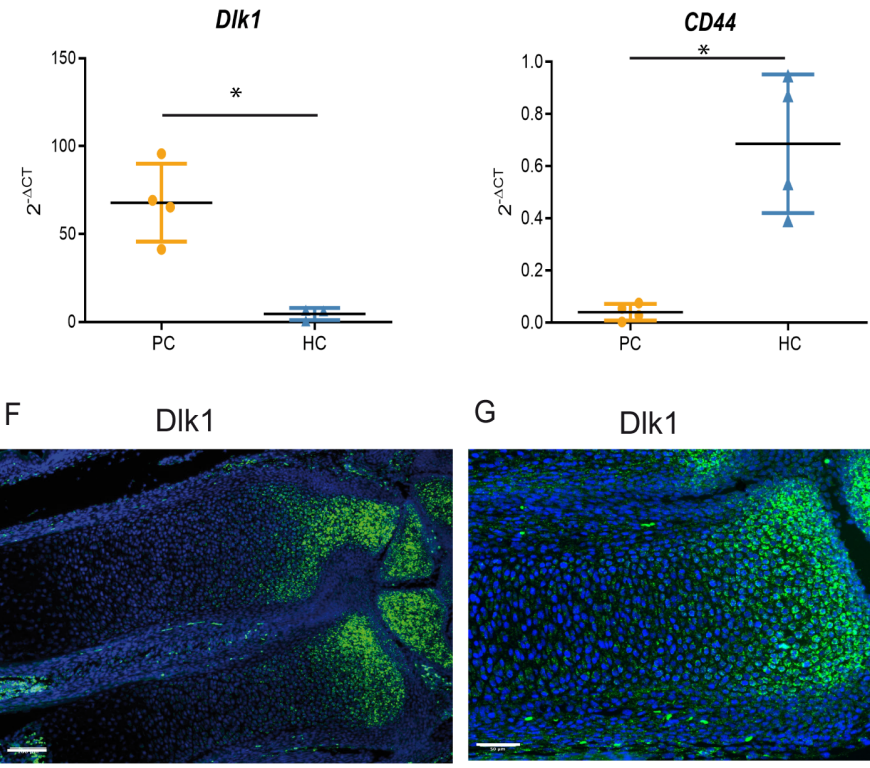

G Dlk1
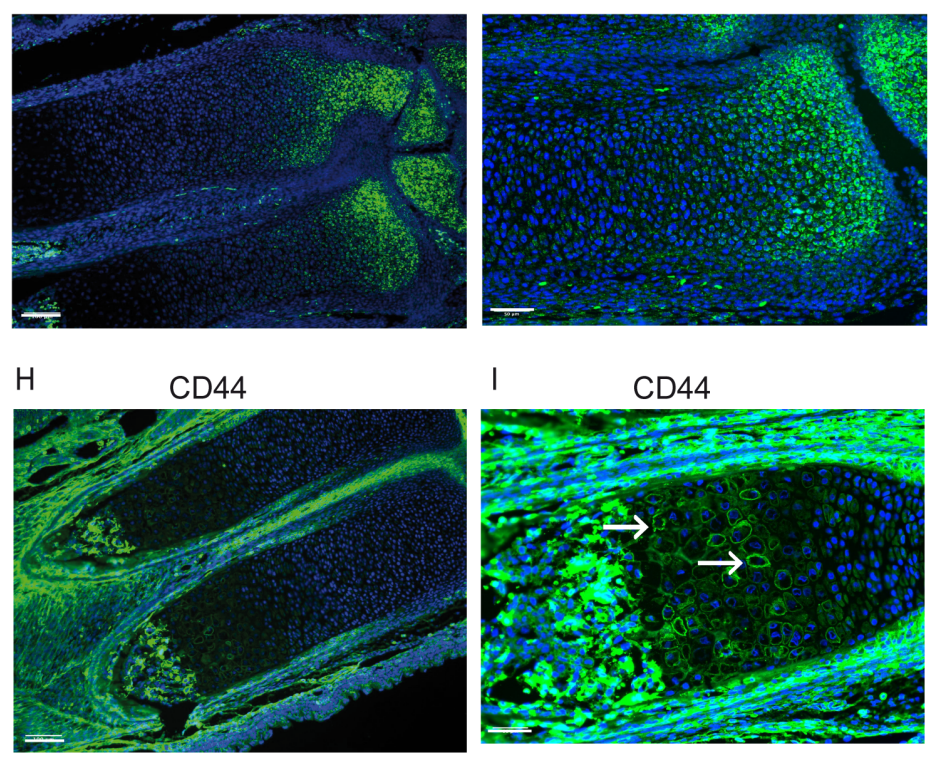

I $\quad$ CD44

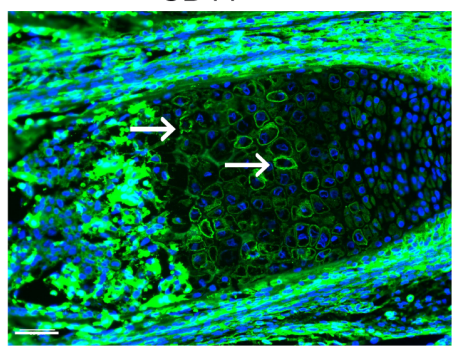

Fig 4. Legend on next page.

by both cell types. Assignment of SEs to the nearest gene identified tissue-specific SEs associated with regulators of chondrogenesis, like Sox5, Sox9, and Col10a1, besides new potential regulators (Supplemental Table S6). We identified 30 genes, including Tcf4 and Smad7, that carried cell type-specific SEs at different genomic locations indicating different activating mechanisms in the respective cell type (Supplemental Table S6). Enrichment analysis assigned similar biological functions to SEs as to distal enhancer states, chondrogenic differentiation for PC-specific SEs, and metabolism for HC-specific SEs 
(Supplemental Fig. S8A). On average, genes carrying a cell typespecific SE were expressed at a significantly higher level in the respective cell population (Fig. 5G, $\mathrm{H}$; Supplemental Fig. S8B) with several PHC-specific genes being associated with PCspecific SEs (Supplemental Table S6). Comparing the relation of SEs to independently obtained expression data ${ }^{(7)}$ confirmed the association of PC-specific SEs to genes expressed in PC and $\mathrm{PHC}$, whereas HC-specific SEs were mainly associated with genes found in the lower hypertrophic zone (Fig. 5/).

To gain insight into the transcriptional control of chondrocyte differentiation, we screened the enhancer states for predicted transcription factor binding motifs. We found binding motifs for Maz, Max, Srf/Fos, Runx, Ets1, C/EBP, and Tead, general regulators of differentiation, differentially enriched in both cell types (Supplemental Fig. S7E). In accordance with their role as main regulators of differentiation, SEs included additional binding sites for chondrogenesis-related transcription factors like Sox9, LEF/TCF, FoxD1, FoxA1, and GATA in PC and NFkb and Pax2 in HC (Fig. 5F).

As developmentally relevant transcription factors frequently bind to less-conserved binding motifs, ${ }^{(17,64)}$ we compared Sox9 ChIP-seq data ${ }^{(17)}$ with our enhancer states. Sox9 peaks have been classified as being located close to the TSS ( \pm 500 bp; class I), regulating general cellular functions, or outside this region (>500 bp; class II) controlling the differentiation of the chondrogenic lineage. ${ }^{(17)}$ As expected, proximal enhancers merely overlapped with Sox9 peaks of either class. The distal enhancers contained few class I peaks, whereas $7 \%$ to $20 \%$ of the distal enhancer overlapped with class II peaks. Intriguingly, nearly all SEs colocalized with Sox9 class II peaks, whereas $10 \%$ to $25 \%$ contained class I peaks (Fig. 6A, B; Supplemental Fig. S8C). In accordance with the collection of mainly $\mathrm{PC}$ and $\mathrm{PHC}$ in the study by Ohba and colleagues, ${ }^{(17)}$ common and PC-specific SEs showed the highest overlap with either class of Sox9 peaks (Fig. 6A). Together, these results support the hypothesis that establishing the Sox9-dependent chondrogenic fate is the main biological function of PC, whereas the differentiation of $\mathrm{HC}$ is dependent on Sox9-independent metabolic pathways.

\section{Cell state transitions are associated with a rapid change in enhancer usage}

Enhancers have been predicted to act on genes within the same topologically associating domain (TAD) ${ }^{(65)}$ with the number of H3K27ac peaks within a TAD correlating with the strength and the robustness of gene expression. ${ }^{(38,39)}$ To gain further insight into the importance of enhancer coverage on gene expression, we calculated the number of $\mathrm{H} 3 \mathrm{~K} 27 \mathrm{ac}$ peaks within a TAD ${ }^{(38)}$ and ranked TADs according to the ratio of $\mathrm{H} 3 \mathrm{~K} 27 \mathrm{ac}$ peaks between PC and HC (Fig. 6C, D; Supplemental Fig. S8D, E; Supplemental Table S7). The TADs with the greatest differential number of H3K27ac peaks contained the genes Sox5, Sox9, and Col10a1. Other TADs with high differences in enhancer marks contained transcriptional regulators like Fox-family proteins in PC and Tead1 in HC (Fig. 6D; Supplemental Fig. S8E; Supplemental Table S7) marking them as potentially important regulators of chondrocyte differentiation. In agreement with the decline in Sox 9 and Sox5 expression during hypertrophy, the clustering of enhancer peaks observed in PC was lost in $\mathrm{HC}$, but few $\mathrm{H} 3 \mathrm{~K} 27 \mathrm{ac}$ peaks were maintained (Fig. $6 E, F$ ). In contrast, the TAD covering the Col10a1 gene contained few H3K27ac peaks in PC, whereas their number was increased, and several clusters were identified in HC (Fig. 6G). Several of the Sox5 and Sox9 associated enhancers matched conserved enhancers of the Vista database, but none of the HC-specific enhancers were included (Fig. 6E-G), indicating that less conserved mechanisms regulate hypertrophy. To test the correlation of peak accumulation and expression changes, we inspected the genomic location of genes like Fos, Gadd45b, and Rxra, which were upregulated and linked to SEs in HC (Fig. 6H-J). As observed for Col10a1, the genomic regions were premarked with $\mathrm{H} 3 \mathrm{~K} 27 \mathrm{ac}$ peaks in $\mathrm{PC}$, pointing to a priming of enhancer clusters before gene expression and a fast accumulation of additional marks with the onset of expression.

\section{Discussion}

Previous studies of chromatin remodeling focused mainly on the in vitro differentiation of embryonic and mesenchymal stem cells into different cell lineages, ${ }^{(1-3)}$ but the epigenetic mechanisms regulating distinct differentiation steps within one cell type are less well understood. In this study, we have, for the first time, investigated changes in the epigenetic profile linked to a specific differentiation step, the terminal differentiation of proliferating into hypertrophic chondrocytes, and have correlated these to differential gene expression.

Analyzing a set of activating and repressive histone marks, we generated an unbiased map of epigenetic modifications. Interestingly for $\mathrm{H} 3 \mathrm{~K} 4 \mathrm{me} 3, \mathrm{H} 3 \mathrm{~K} 9 \mathrm{ac}$, and $\mathrm{H} 3 \mathrm{~K} 27 \mathrm{ac}$, not only the coverage but also the differential level of modification correlated with differences in gene expression, identifying them as predictors for changes in transcriptional activity. H3K27me3 negatively correlated with the activating promoter marks, indicating that its differential occupancy also affects gene expression. Combining histone marks into states by ChromHMM identified two states that combine the repressive mark $\mathrm{H} 3 \mathrm{~K} 27$ me3 with either activating promoter marks (state 13) or H3K36me3 (state 15). Analyzing the frequency of state transitions between $\mathrm{PC}$ and $\mathrm{HC}$ in a set of

Fig 4 Integration of RNA-seq and ChIP-seq data. (A) Chondrocyte-specific genes with the transition from the active promoter state 9 to the repressedactive promoter state 13 (blue), from the TSS transcribed gene state 11 to the repressed-active promoter state 13 (orange), or both transitions (black) were sorted by their expression level (beta value) and assigned to their published expression pattern in PC, PHC and HC, or not annotated (NA). Representative genes are depicted. (B) Model depicting differences in chondrocyte isolation for ChIP-seq and RNA-seq. PHCs were enriched in the PC pool for ChIP-seq and in the $\mathrm{HC}$ pool for RNA-seq. (C) Number of genes in a given Pareto front with front 1 being the optimal. Genes are color coded according to their differential expression. Orange: $\mathrm{PC}>\mathrm{HC}$; blue: $\mathrm{HC}>\mathrm{PC}$. Representative genes of the topmost- and lowest-ranking Pareto fronts are labeled. (D, $E$ ) Differential expression of Dlk1 $(D)$ and CD44 (E) mRNA was measured by qPCR $\left(2^{-\triangle C T}\right)$ detecting higher expression of Dlk1 in PC (orange) and CD44 in HC (blue). $n=4$ biological replicates; $p=0.0013(D), p=0.022(E)$, error bars show standard deviation. $(F, G)$ In situ hybridization of Dlk1 on sections of E16.5 forelimbs detects expression in PC. $(H, I)$ Immunofluorescence on E16.5 forelimb sections detects CD44 protein in the cell membrane of HC (white arrows), the periosteum, and the bone marrow. Nuclei were counterstained with DAPI. Scale bars: $100 \mu \mathrm{m}(F, H) ; 50 \mu \mathrm{m}(G, I)$. 
A

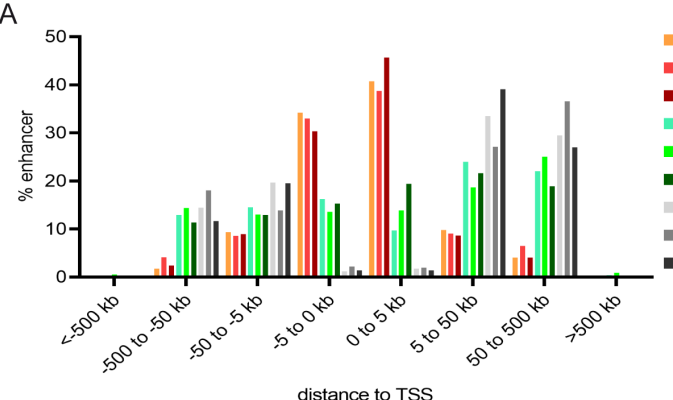

C
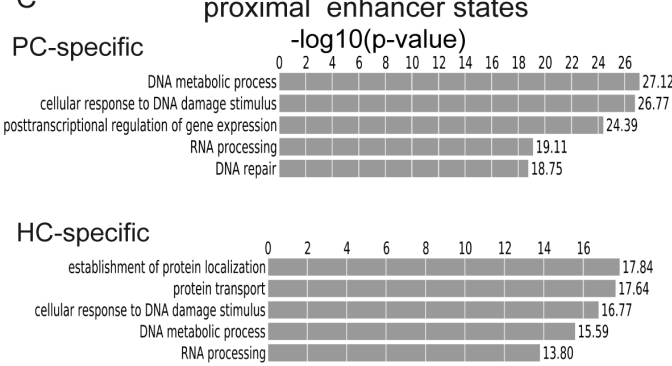

common

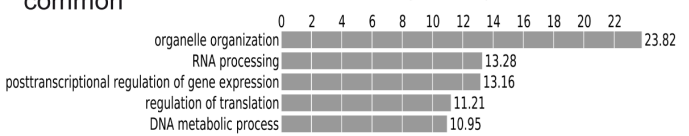

E

\begin{tabular}{|r|c|c|c|}
\hline limb E13.5 & 78.25 & 39.28 & 25.71 \\
\hline limb E15.5 & 88.13 & 45.77 & 41.74 \\
heart E13.5 & 83.57 & 35.90 & 41.62 \\
\hline heart E15.5 & 84.13 & 32.26 & 41.54 \\
\hline liver E13.5 & 71.80 & 18.66 & 26.79 \\
\hline liver E15.5- & 62.92 & 10.59 & 17.43 \\
\hline neural tube E13.5- & 78.49 & 30.45 & 26.83 \\
\hline neural tube E15.5- & 78.79 & 26.78 & 24.27 \\
\hline forebrain E13.5- & 76.51 & 30.54 & 28.57 \\
\hline forebrain E15.5- & 70.99 & 21.60 & 19.68 \\
\hline bone marrow 8w- & 60.17 & 9.08 & 15.04 \\
\hline heart 8w- & 63.48 & 16.89 & 24.72 \\
\hline liver 8w- & 57.27 & 10.28 & 15.99 \\
\hline cerebellum 8w- & 52.32 & 10.74 & 10.81 \\
\hline
\end{tabular}

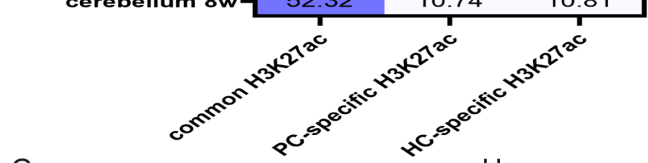

G

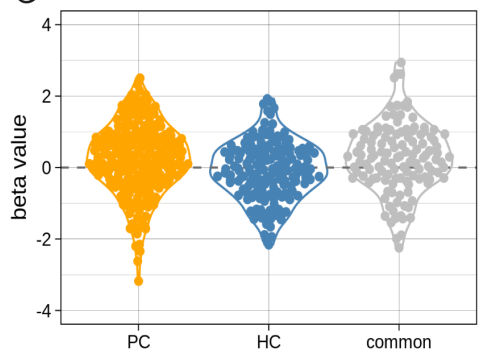

$\mathrm{H}$

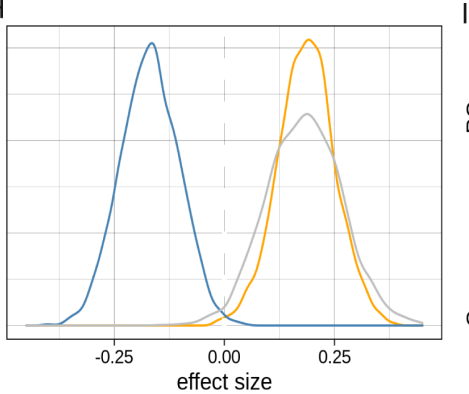

B

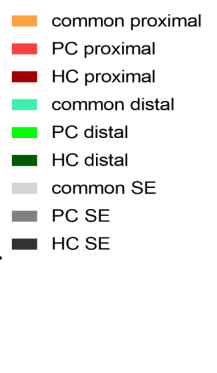

$\mathrm{D}$

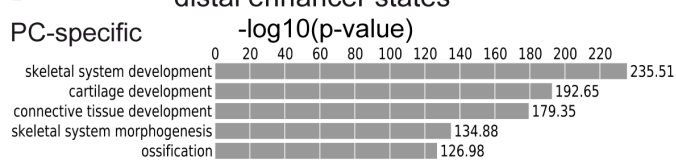

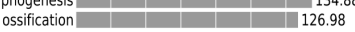

HC-specific

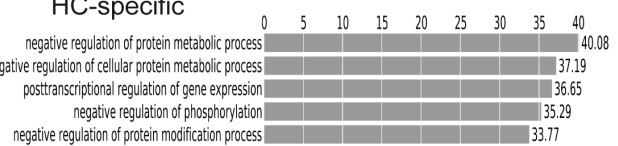

common

\begin{tabular}{l|llllllllllllll} 
& 0 & 5 & 10 & 15 & 20 & 25 & 30 & 35 & 40 & 45 & 50 & 55 & 60 & \\
cardiovascular system development & & & & & & & & & & & & & &
\end{tabular}

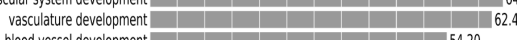
\begin{tabular}{|l|l|l|l|l|l|}
\hline blood vessel development & & & 54.20 \\
\hline
\end{tabular} development ending in birth or egg hatching $\quad$. 51.39

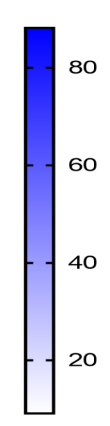

\section{$\mathrm{F}$}

$-\log 10$ p-value

$1 \quad 1 \mathrm{E}+10 \quad 1 \mathrm{E}+20 \quad 1 \mathrm{E}+30 \quad 1 \mathrm{E}+40$
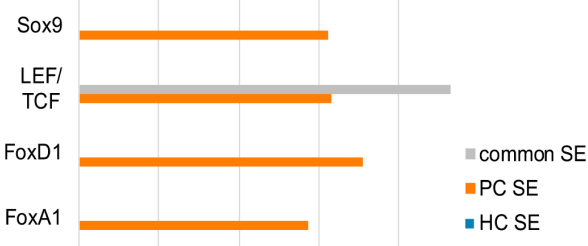

GATA

NFkb

Pax2
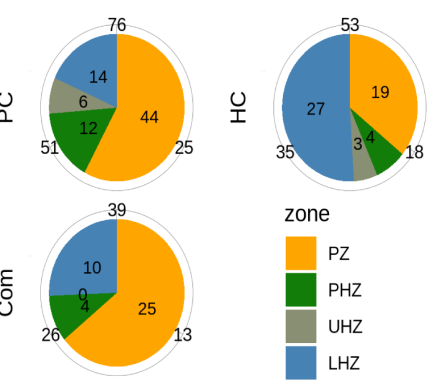

Fig 5. Legend on next page.

chondrogenesis-related genes revealed a specific enrichment of transitions from activating promoter states 9 and 11 into the repressed-active promoter state 13 on genes like Ptch1, Sox5, Sox6, and Sox9, which are downregulated during hypertrophy. Furthermore, integrating differential expression and epigenetic modifications identified many genes known to be downregulated in $\mathrm{HC}$ in the topmost-ranking Pareto fronts, supporting the hypothesis that the addition of $\mathrm{H} 3 \mathrm{~K} 27 \mathrm{me} 3$ on regions still marked as active and not the removal of activating marks is the repression-inducing event.

Overall, the differential occupancy of promoter marks correlated well with gene expression. In contrast, the lowest-ranking 
Pareto fronts displayed a specific discrepancy between activating marks and gene expression. These fronts include critical regulators of cell cycle exit and hypertrophy, like Pth1r and Runx2, which are specifically upregulated in $\mathrm{PHC}$ and repressed during hypertrophy. Because the systematic enrichment of these genes cannot be explained by random fluctuation, this discrepancy is likely attributable to differences in cell isolation. Cells were isolated based on genetically induced fluorescence for ChIP-seq, which led to the enrichment of PHCs in the PC pool, as demonstrated by the increased decoration of activating marks on PHC-specific genes. In contrast, based on gene expression detected by RNA-seq, PHCs were clearly enriched in the $\mathrm{HC}$ pool, which was isolated based on morphology. Consequently, the chosen isolation strategy led to a discrepancy between RNA expression and the histone profile, leading to the specific accumulation of PHC-specific genes in the lowest-ranking Pareto fronts. These fronts will serve as an important source to identify novel regulators of the transition into hypertrophy.

A gain of $\mathrm{H} 3 \mathrm{~K} 27 \mathrm{me} 3$ at $\mathrm{H} 3 \mathrm{~K} 4 \mathrm{me} 3$-decorated promoters has recently been described for neuronal genes downregulated in the process of neural differentiation. ${ }^{(66)}$ The presence of other activating marks, which were included in our data, has not been investigated. It is likely that during differentiation, neuronal progenitor cells, like chondrocytes, add H3K27me3 to promoters marked as active by other modifications besides H3K4me3. Sodersten and colleagues ${ }^{(66)}$ also detected an enrichment of the combination $\mathrm{H} 3 \mathrm{~K} 27 \mathrm{me} 3 / \mathrm{H} 3 \mathrm{~K} 9 \mathrm{me} 3$ (corresponding to our state 1 ; silenced) on genes that are permanently silenced in differentiated neurons. In our data set, we found an enrichment of the transitions 1.13 (silenced/repressed-active promoter) and 13.1. These transitions were mostly associated with early limb patterning genes, which are no longer expressed in either chondrocyte type and likely reflect the transition into permanent repression with rare leftovers of activating marks. Surprisingly, genes downregulated between PC and HC were not enriched in the transition into the silenced state 1 . This difference to the neuronal data likely reflects the longevity of neurons, whereas HCs have a life span of a few days before they undergo apoptosis or transdifferentiate into osteoblasts. ${ }^{(67,68)}$ They might thus not have sufficient time to convert the epigenetic decoration from repressed-active into permanently silenced. In neuronal cells, genes repressed by $\mathrm{H} 3 \mathrm{~K} 4 \mathrm{me} 3 / \mathrm{H} 3 \mathrm{~K} 27 \mathrm{me} 3$ are preferably reactivated during neuronal stress. ${ }^{(66)}$ Recent studies demonstrated that hypertrophic chondrocytes undergoing ER stress can reenter the proliferating state. ${ }^{(69,70)}$ One might thus speculate that the incomplete loss of activating marks facilitates this redifferentiation under pathological conditions.
Whereas the repression of genes is linked to the repressedactive chromatin modification, we did not detect an enrichment in transitions from a bivalent to an activating state in genes upregulated in $\mathrm{HC}$ as it has been observed for lineage-specific genes in ES cells. ${ }^{(71)}$ Inspection of CD44, which we identified as a new maker of $\mathrm{HC}$, did not show repressive marks at the promoter region in PC. The bivalent modification preparing for expression might thus be preferentially found in ES cells, ${ }^{(71)}$ whereas at later developmental stages, lineage-specific genes have lost the pluripotency associated label. In summary, although we did not find an enrichment of repressed-active modifications on genes prone to be upregulated in $\mathrm{HC}$, we gained strong evidence that the initiation of gene repression is linked to the addition of the repressive mark H3K27me3 to regions still marked as active, identifying the addition of this mark rather than the removal of activating marks as the repressioninducing event. Long-term repression might subsequently require the addition of $\mathrm{H} 3 \mathrm{~K} 9 \mathrm{me} 3$ as indicated by the neuronal data.

The modification of histone marks is regulated by a large number of enzymes. Many of these are expressed in chondrocytes; however, their global epigenetic role in the process of chondrocytes differentiation has not been systematically investigated. Nevertheless, inactivation of components of the polycomb repressive complex (PRC), which mediate the trimethylation of $\mathrm{H} 3 \mathrm{~K} 27$, led to distinct skeletal malformations, ${ }^{(72)}$ whereas chondrocyte-specific deletion of Ezh2, the enzymatic component of PRC, had no obvious effect on growth plate morphology. ${ }^{(73)}$ Surprisingly, deletion of the H3K27me3 demethylase Jmjd3 also disturbs chondrocyte morphology, ${ }^{(74)}$ indicating that $\mathrm{H} 3 \mathrm{~K} 27$ trimethylation needs to be tightly balanced to ensure the timely onset of hypertrophy. In this context, it is also noteworthy that several transcriptional regulators, like Sox9, Mef2c, and Runx2, interact with histonemodifying enzymes and recruit them to specific transcriptional targets, ${ }^{(75,76)}$ thereby providing an additional level of activation and specificity. In summary, while first mouse mutants support the importance of histone-modifying enzymes for chondrocyte differentiation, deciphering their exact function will require extensive systematic analyses of changes in the epigenetic profile and the interaction with the transcription machinery. Furthermore, repressive histone modifications are tightly linked to the methylation of DNA. Although changes in the DNA methylation status have been associated with hypertrophic differentiation of articular chondrocytes, ${ }^{(77-79)}$ a systematic analysis in distinct chondrocytes subtypes is yet lacking. Correlating DNA methylation to H3K27me3 decoration at promoters will subsequently be required to fully understand epigenetic alterations initiating gene repression in the chondrogenic lineage.

Fig 5 Characterization of enhancers in PC and HC. (A) Distribution of proximal and distal enhancer states and SEs in PC, HC, or both (common) at a given distance from the TSS of the assigned gene. (B) The percentage of enhancer states and SEs more than $500 \mathrm{~kb}$ distant from the assigned gene. (C, D) Enrichment analysis of genes annotated to either proximal $(C)$ or distal $(D)$ enhancer states showing the five GO terms with the highest enrichment for PC-specific, HC-specific, and common enhancers. Enhancers were assigned to the nearest gene within $1000 \mathrm{~kb}$ using GREAT. (E) Comparison of common and cell type-specific H3K27ac peaks to H3K27ac data sets of different embryonic and postnatal tissues. (F) Enrichment (-log10 $p$ value) of predicted transcription factor binding sites. (G-l) Differential gene expression $(G)$ and effect size of the correlation $(H)$ between genes regulated by PC-specific, HC-specific, and common SEs compared with the differential expression of all genes. (I) Pie chart of the distribution of SE-carrying genes in PC, HC, or both cell types (com$\mathrm{mon}$ ) in relation to their published expression pattern in the proliferating (PZ), prehypertrophic (PHZ), upper hypertrophic (UHZ) and lower hypertrophic (LHZ) zone. ${ }^{(7)}$ The total number of enhancers in the specified zone and cell type is given. (F-l) Orange: PC-specific SE; blue: HC-specific SE; gray: common. 
A

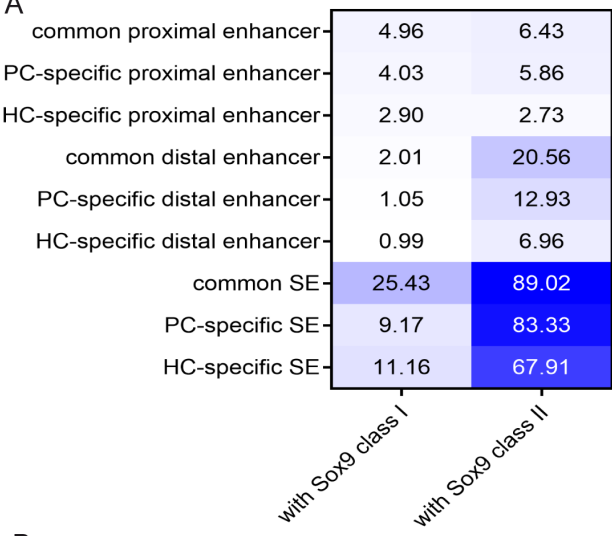

B Effect size of Sox9 and SE overlap
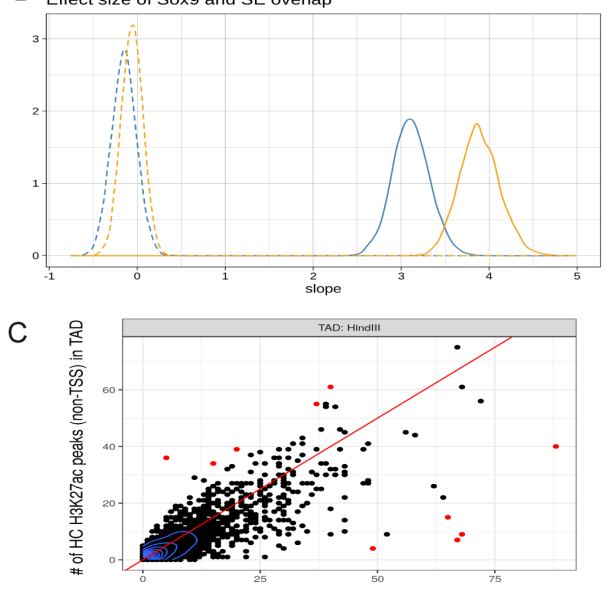

\# of PC H3K27ac peaks (non-TSS) in TAD

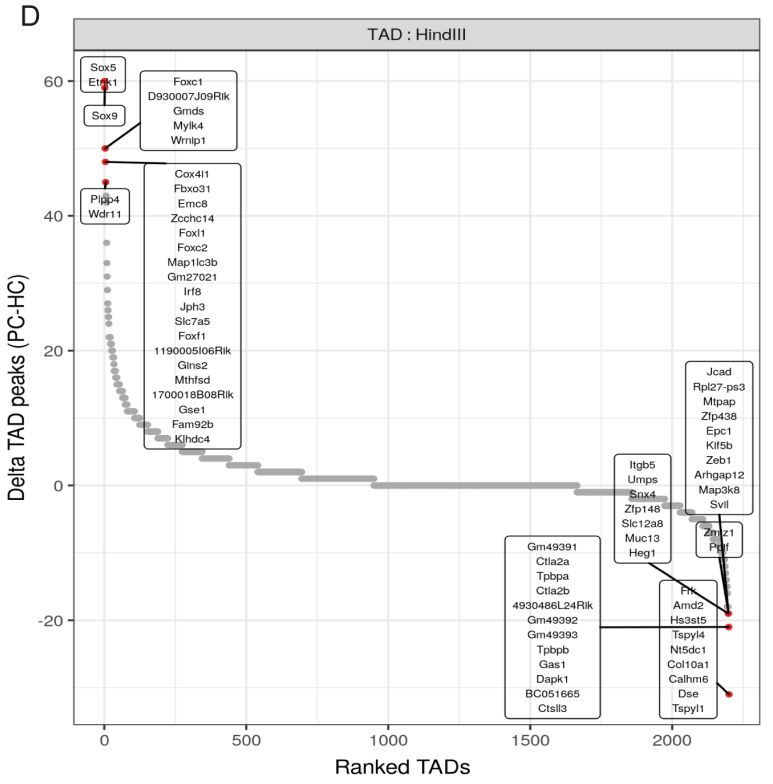

E

80

60

40

20

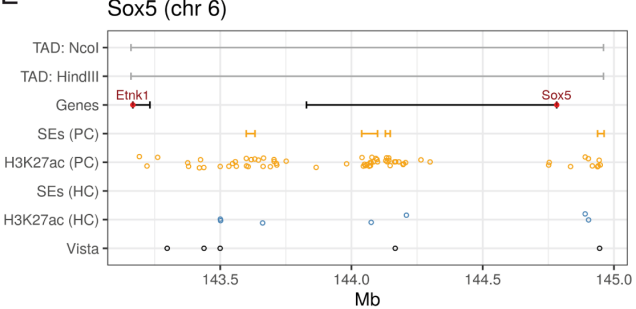

F

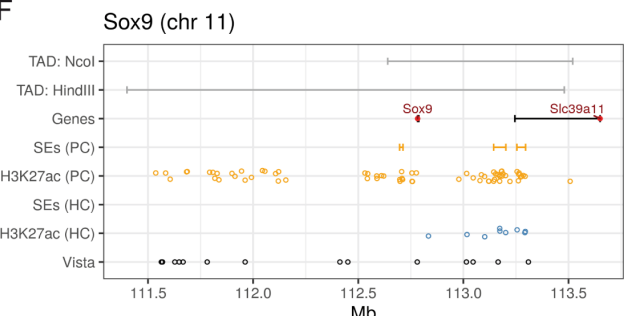

G

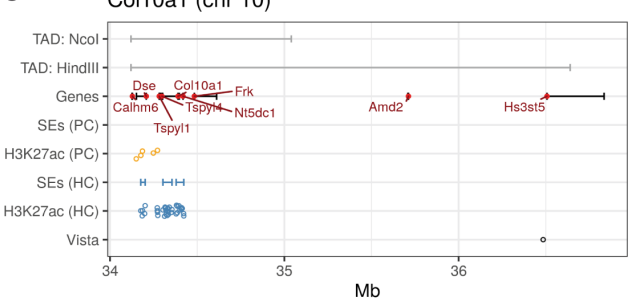

$\mathrm{H}$
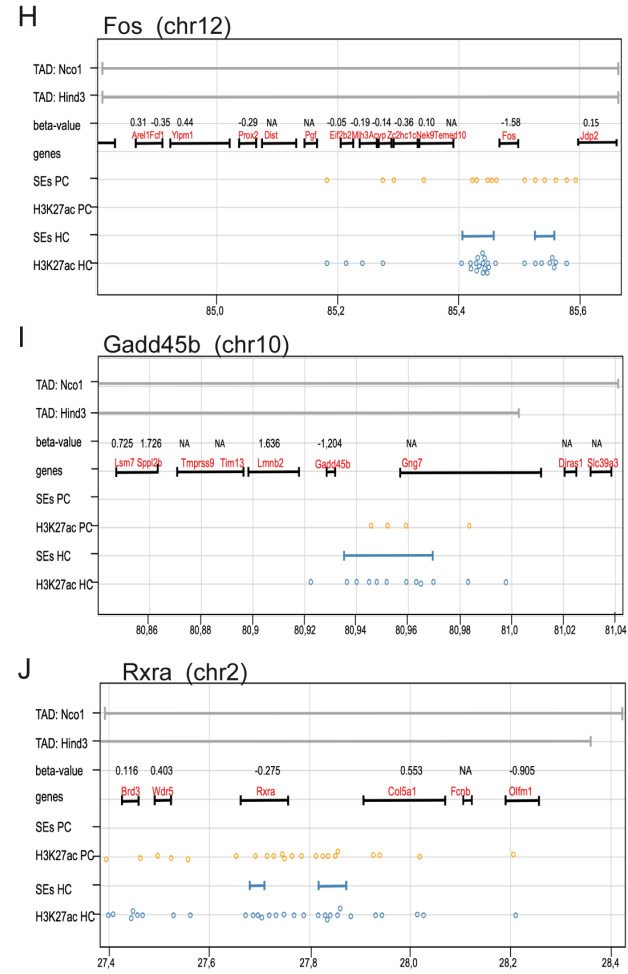

Fig 6. Correlation of differential H3K27ac occupancy within a TAD with cell type-specific gene expression. (A) Colocalization of different enhancer states and SEs in PC and HC with class I and class II Sox9 peaks. (B) Effect size of Sox9 peak enrichment in SEs in both cell types compared with randomly chosen genomic regions of similar size. (C) The number of H3K27ac peaks outside the TSS in a TAD in PC ( $x$-axis) and HC ( $y$-axis) was plotted. Red dots show TADs with the highest number of differential H3K27ac peaks between PC and HC (compare to deltaTADs in $D$ ). (D) TADs were ranked according to their differential occupancy with H3K27ac peaks (deltaTAD peaks). Genes in the highest- and lowest-ranking TADs are given. (E-l) H3K27ac peaks (circles) and SEs (bars) detected in PC (orange) and HC (blue) within TADs (gray bars) containing Sox5 $(E)$, Sox9 $(F)$, Col10a1 $(G)$, Fos $(H)$, Gadd45b $(I)$, and Rxra $(J)$ are given with the expression of all genes in the respective TAD. Distances are given in Mb. 
To gain further insight into the cell state-specific control of gene expression, we identified enhancers based on H3K27ac occupancy. Although both PC and HC belong to the chondrogenic lineage, the identified enhancers are quite different, with only about $30 \%$ common to both cell types. GO analyses gave insight into the chondrocyte-specific function of enhancer associated genes. As expected, PC-specific enhancers were enriched in chondrogenesis-related pathways establishing the cell lineages. Furthermore, a high percentage contained a Sox9 class II binding site that has been shown to regulate chondrogenic genes. ${ }^{(17)}$ Intriguingly, continuous activation of lineage-defining genes is less important in $\mathrm{HC}$ after the chondrogenic lineage has been established. The loss of lineage-specific enhancers might be indicative for the terminal differentiation of a cell type. In contrast to PC, HC-specific enhancers related mainly to metabolismassociated genes. Little is known about the mechanisms by which HCs acquire their size and specific ECM. Increased metabolic activity, ${ }^{(80,81)}$ Bmp-dependent mTor, ${ }^{(82,83)}$ and IGF1 signaling ${ }^{(84,85)}$ have been implicated in regulating hypertrophy, and our data strongly emphasize the importance of metabolism for the generation of HC. Moreover, analyzing the conservation of enhancers strongly indicates that the switch to general cellular and metabolic function might be characteristic for terminal differentiated tissues.

The association of enhancers to the regulated gene is hampered by their function over long distances, which may also include other genes. ${ }^{(19,86,87)}$ It is thus difficult to predict if specific enhancers control different genes or if different enhancers regulate the same gene at distinct differentiation stages. Inspection of SEs indicates that both might be the case. Differentially expressed genes, like Sox9 and Col10a1, were associated with cell type-specific SEs, whereas genes like Tcf4 and Smad7 were assigned to different SEs in PC and HC. More direct investigations of enhancer usage by, for example, unbiased chromatin conformation capture ${ }^{(88)}$ will be required to gain a detailed understanding of their cell type-specific use, interaction, and function.

Several studies have linked the number of enhancers to the level and robustness of gene expression. ${ }^{(89)}$ To analyze differences in enhancer usage, we ranked TADs by their differential H3K27ac abundance. The highest-ranking TADs contained cell type-specific genes like Sox9, Sox5, and Col10a1, whereas genes like Smad7 and Tcf4, which are regulated by distinct SEs in either cell type, were found in average-ranked TADs. Inspection of specific TADs confirmed that the downregulation of genes like Sox9 and Sox5 was linked to a rapid loss of enhancers in $\mathrm{HC}$. In contrast, upregulation of genes like Col10a1 and Gadd45b in HC was accompanied by a low enhancer coverage in PC followed by a dramatic increase in HC. Although the association of enhancers to genes was only possible for selected TADs, correlating expression differences of single genes to enhancer usage inside a TAD in more detailed studies will provide valuable insight into the control of gene expression. ${ }^{(90,91)}$ Furthermore, recent studies indicate that TADs might be remodeled depending on transcription. ${ }^{(65)}$ Establishing chondrocyte-specific TADs will, thus, refine the interpretation of enhancer association and usage. In summary, although differences in enhancer usage have been reported for genes expressed in different cell lineages, ${ }^{(92)}$ our data provide first insight into the dynamics of enhancer usage in sequential differentiation stages.

\section{Disclosures}

All authors state that they have no conflicts of interest.

\section{Acknowledgments}

The authors thank Sabine Schneider for excellent technical assistance and critical reading of the manuscript and Dr Klein-Hitpass for sequencing data. This work was supported by the DFG research unit ExCarBon (FOR 2407; Vo620/14) and the DFG graduate program GRK 1431 to AV.

Author contributions: Manuela Wuelling: Conceptualization; formal analysis; investigation; methodology; validation; visualization; writing-original draft; writing-review \& editing. Christoph Neu: Formal analysis; investigation; methodology; software; visualization; writing-review \& editing. Andrea Thiesen: Investigation; methodology. Simo Kitanovski: Formal analysis; methodology; software; validation; visualization. Yingying Cao: Formal analysis; methodology; software; validation; visualization. Anja Lange: Formal analysis; methodology; software; validation. Astrid Westendorf: Resources. Daniel Hoffmann: Software; supervision; writing-review \& editing. Andrea Vortkamp: Conceptualization; funding acquisition; project administration; supervision; writing-original draft; writing-review \& editing.

\section{PEER REVIEW}

The peer review history for this article is available at https:// publons.com/publon/10.1002/jbmr.4263.

\section{References}

1. Mikkelsen TS, Ku M, Jaffe DB, et al. Genome-wide maps of chromatin state in pluripotent and lineage-committed cells. Nature. 2007;448 (7153):553-560.

2. Wu H, Gordon JA, Whitfield TW, et al. Chromatin dynamics regulate mesenchymal stem cell lineage specification and differentiation to osteogenesis. Biochim Biophys Acta Gene Regul Mech. 2017;1860 (4):438-449.

3. Herlofsen SR, Bryne JC, Hoiby T, et al. Genome-wide map of quantified epigenetic changes during in vitro chondrogenic differentiation of primary human mesenchymal stem cells. BMC Genomics. 2013; $14: 105$.

4. Bradley EW, McGee-Lawrence ME, Westendorf JJ. Hdac-mediated control of endochondral and intramembranous ossification. Crit Rev Eukaryot Gene Expr. 2011;21(2):101-113.

5. Kozhemyakina E, Lassar AB, Zelzer E. A pathway to bone: signaling molecules and transcription factors involved in chondrocyte development and maturation. Development. 2015;142(5):817-831.

6. Wuelling $M$, Vortkamp A. Chondrocyte proliferation and differentiation. Endocr Dev. 2011;21:1-11.

7. Tan Z, Niu B, Tsang KY, et al. Synergistic co-regulation and competition by a SOX9-GLI-FOXA phasic transcriptional network coordinate chondrocyte differentiation transitions. PLoS Genet. 2018;14(4): e1007346.

8. Guenther MG, Levine SS, Boyer LA, Jaenisch R, Young RA. A chromatin landmark and transcription initiation at most promoters in human cells. Cell. 2007;130(1):77-88.

9. Wagner EJ, Carpenter PB. Understanding the language of Lys36 methylation at histone H3. Nat Rev Mol Cell Biol. 2012;13(2):115-126.

10. Volpe TA, Kidner C, Hall IM, Teng G, Grewal SI, Martienssen RA. Regulation of heterochromatic silencing and histone $\mathrm{H} 3$ lysine- 9 methylation by RNAi. Science. 2002;297(5588):1833-1837.

11. Zhang T, Cooper S, Brockdorff N. The interplay of histone modifications - writers that read. EMBO Rep. 2015;16(11):1467-1481. 
12. Li F, Wan M, Zhang B, et al. Bivalent histone modifications and development. Curr Stem Cell Res Ther. 2018;13(2):83-90.

13. Alder O, Lavial F, Helness A, et al. Ring1B and Suv39h1 delineate distinct chromatin states at bivalent genes during early mouse lineage commitment. Development. 2010;137(15):2483-2492.

14. Bernstein BE, Mikkelsen TS, Xie X, et al. A bivalent chromatin structure marks key developmental genes in embryonic stem cells. Cell. 2006; 125(2):315-326.

15. Hahn MA, Wu X, Li AX, Hahn T, Pfeifer GP. Relationship between gene body DNA methylation and intragenic H3K9me3 and H3K36me3 chromatin marks. PLoS One. 2011;6(4):e18844.

16. Mauser R, Kungulovski G, Keup C, Reinhardt R, Jeltsch A. Application of dual reading domains as novel reagents in chromatin biology reveals a new $\mathrm{H} 3 \mathrm{~K} 9 \mathrm{me} 3$ and $\mathrm{H} 3 \mathrm{~K} 36 \mathrm{me} 2 / 3$ bivalent chromatin state. Epigenetics Chromatin. 2017;10(1):45.

17. Ohba S, He X, Hojo H, McMahon AP. Distinct transcriptional programs underlie Sox9 regulation of the mammalian chondrocyte. Cell Rep. 2015;12(2):229-243.

18. Liu CF, Lefebvre V. The transcription factors SOX9 and SOX5/SOX6 cooperate genome-wide through super-enhancers to drive chondrogenesis. Nucleic Acids Res. 2015;43(17):8183-8203.

19. Guo M, Liu Z, Willen J, et al. Epigenetic profiling of growth plate chondrocytes sheds insight into regulatory genetic variation influencing height. Elife. 2017;6:e29329.

20. Richard D, Liu Z, Cao J, et al. Evolutionary selection and constraint on human knee chondrocyte regulation impacts osteoarthritis risk. Cell. 2020;181(2):362-381.

21. Terpstra L, Prud'homme J, Arabian A, et al. Reduced chondrocyte proliferation and chondrodysplasia in mice lacking the integrin-linked kinase in chondrocytes. J Cell Biol. 2003;162(1): 139-148.

22. Gebhard S, Hattori T, Bauer E, et al. Specific expression of Cre recombinase in hypertrophic cartilage under the control of a BAC-Col10a1 promoter. Matrix Biol. 2008;27(8):693-699.

23. Srinivas $S$, Watanabe T, Lin CS, et al. Cre reporter strains produced by targeted insertion of EYFP and ECFP into the ROSA26 locus. BMC Dev Biol. 2001;1:4.

24. Andrews S. FastQC: a quality control tool for high throughput sequence data. 2010. Available online at: http://www. bioinformatics.babraham.ac.uk/projects/fastqc.

25. Schmieder R, Edwards R. Quality control and preprocessing of metagenomic datasets. Bioinformatics. 2011;27(6):863-864.

26. Li H, Durbin R. Fast and accurate short read alignment with burrowswheeler transform. Bioinformatics. 2009;25:1754-1760.

27. Kharchenko PV, Tolstorukov MY, Park PJ. Design and analysis of ChIPseq experiments for DNA-binding proteins. Nat Biotechnol. 2008;26: 1351-1359.

28. Okonechnikov K, Conesa A, García-Alcalde F. Qualimap 2: advanced multi-sample quality control for high-throughput sequencing data. Bioinformatics. 2016;32:292-294.

29. Landt SG, Marinov GK, Kundaje A, et al. ChIP-seq guidelines and practices of the ENCODE and modENCODE consortia. Genome Res. 2012; 22(9):1813-1831.

30. Lawrence $M$, Huber W, Pagès $H$, et al. Software for computing and annotating genomic ranges. PLoS Comput Biol. 2013;9:e1003118.

31. Lun ATL, Smyth GK. Csaw: a bioconductor package for differential binding analysis of ChIP-seq data using sliding windows. Nucleic Acids Res. 2016;44:e45.

32. Starmer J, Magnuson T. Detecting broad domains and narrow peaks in ChIP-seq data with hiddenDomains. BMC Bioinformatics. 2016; 17:144.

33. Robinson JT, Thorvaldsdottir $\mathrm{H}$, Winckler $\mathrm{W}$, et al. Integrative genomics viewer. Nat Biotechnol. 2011;29(1):24-26.

34. Ernst J, Kellis M. ChromHMM: automating chromatin-state discovery and characterization. Nat Methods. 2012;9(3):215-216.

35. de Jonge HJ, Fehrmann RS, de Bont ES, et al. Evidence based selection of housekeeping genes. PLoS One. 2007;2(9):e898.
36. Burkner PC. Advanced Bayesian multilevel modeling with the R package brms. R J. 2018;10(1):395-411.

37. Huang J, Li K, Cai W, et al. Dissecting super-enhancer hierarchy based on chromatin interactions. Nat Commun. 2018;9(1):943.

38. Dixon JR, Selvaraj S, Yue F, et al. Topological domains in mammalian genomes identified by analysis of chromatin interactions. Nature. 2012;485(7398):376-380.

39. Hnisz D, Abraham BJ, Lee TI, et al. Super-enhancers in the control of cell identity and disease. Cell. 2013;155(4):934-947.

40. Whyte WA, Orlando DA, Hnisz D, et al. Master transcription factors and mediator establish super-enhancers at key cell identity genes. Cell. 2013;153(2):307-319.

41. McLean CY, Bristor D, Hiller M, et al. GREAT improves functional interpretation of cis-regulatory regions. Nat Biotechnol. 2010;28(5): 495-501.

42. Hung JH, Weng Z. Mapping short sequence reads to a reference genome. Cold Spring Harb Protoc. 2017;2017(2):158-164.

43. Bolger AM, Lohse $M$, Usadel B. Trimmomatic: a flexible trimmer for Illumina sequence data. Bioinformatics. 2014;30(15):2114-2120.

44. Bray NL, Pimentel $H$, Melsted P, Pachter L. Near-optimal probabilistic RNA-seq quantification. Nat Biotechnol. 2016;34(5):525-527.

45. Pimentel H, Bray NL, Puente S, Melsted P, Pachter L. Differential analysis of RNA-seq incorporating quantification uncertainty. Nat Methods. 2017;14(7):687-690.

46. Yu G, Wang LG, Han Y, He QY. clusterProfiler: an R package for comparing biological themes among gene clusters. OMICS. 2012;16(5): 284-287.

47. Shwartz Y, Zelzer E. Nonradioactive in situ hybridization on skeletal tissue sections. Methods Mol Biol. 2014;1130:203-215.

48. Bachvarova V, Dierker T, Esko J, Hoffmann D, Kjellen L, Vortkamp A. Chondrocytes respond to an altered heparan sulfate composition with distinct changes of heparan sulfate structure and increased levels of chondroitin sulfate. Matrix Biol. 2020;93:43-59.

49. Underhill TM, Dranse HJ, Hoffman LM. Analysis of chondrogenesis using micromass cultures of limb mesenchyme. Methods Mol Biol. 2014;1130:251-265.

50. Lev R, Spicer SS. Specific staining of sulphate groups with Alcian blue at low Ph. J Histochem Cytochem. 1964;12:309.

51. Wang C, Wang Z, Chen C, et al. A low MW inhibitor of CD44 dimerization for the treatment of glioblastoma. Br J Pharmacol. 2020;177(13): 3009-3023.

52. Durinck S, Moreau Y, Kasprzyk A, et al. BioMart and bioconductor: a powerful link between biological databases and microarray data analysis. Bioinformatics. 2005;21(16):3439-3440.

53. Durinck S, Spellman PT, Birney E, Huber W. Mapping identifiers for the integration of genomic datasets with the R/bioconductor package biomaRt. Nat Protoc. 2009;4(8):1184-1191.

54. Cao Yingying, Kitanovski Simo, Hoffmann Daniel. intePareto: an R package for integrative analyses of RNA-Seq and ChIP-Seq data. BMC Genomics. 2020;21(S11):802.

55. Li H, Handsaker $B$, Wysoker $A$, et al. The sequence alignment/map format and SAMtools. Bioinformatics. 2009;25:2078-2079.

56. Love Ml, Huber W, Anders S. Moderated estimation of fold change and dispersion for RNA-seq data with DESeq2. Genome Biol. 2014 15(12):550.

57. Iwamoto M, Yagami K, Lu Valle $\mathrm{P}$, et al. Expression and role of c-myc in chondrocytes undergoing endochondral ossification. J Biol Chem. 1993;268(13):9645-9652.

58. Yang $Y$, Topol L, Lee $\mathrm{H}, \mathrm{Wu} J$. Wnt5a and Wnt5b exhibit distinct activities in coordinating chondrocyte proliferation and differentiation. Development. 2003;130(5):1003-1015.

59. Chen L, Qanie D, Jafari A, et al. Delta-like 1/fetal antigen-1 (Dlk1/FA1) is a novel regulator of chondrogenic cell differentiation via inhibition of the Akt kinase-dependent pathway. J Biol Chem. 2011;286(37): 32140-32149.

60. Wang Y, Sul HS. Pref-1 regulates mesenchymal cell commitment and differentiation through Sox9. Cell Metab. 2009;9(3):287-302. 
61. Patti AM, Gabriele A, Vulcano A, Ramieri MT, Della Rocca C. Effect of hyaluronic acid on human chondrocyte cell lines from articular cartilage. Tissue Cell. 2001;33(3):294-300.

62. Sato E, Ando T, Ichikawa J, et al. High molecular weight hyaluronic acid increases the differentiation potential of the murine chondrocytic ATDC5 cell line. J Orthop Res. 2014;32(12):1619-1627.

63. Creyghton MP, Cheng AW, Welstead GG, et al. Histone H3K27ac separates active from poised enhancers and predicts developmental state. Proc Natl Acad Sci U S A. 2010;107(50):21931-21936.

64. Lewandowski JP, Du F, Zhang S, et al. Spatiotemporal regulation of GLI target genes in the mammalian limb bud. Dev Biol. 2015;406(1): 92-103.

65. Rowley MJ, Corces VG. Organizational principles of 3D genome architecture. Nat Rev Genet. 2018;19(12):789-800.

66. Sodersten E, Toskas K, Rraklli V, et al. A comprehensive map coupling histone modifications with gene regulation in adult dopaminergic and serotonergic neurons. Nat Commun. 2018;9(1):1226.

67. Yang L, Tsang KY, Tang HC, Chan D, Cheah KS. Hypertrophic chondrocytes can become osteoblasts and osteocytes in endochondral bone formation. Proc Natl Acad Sci U S A. 2014;111(33):12097-12102.

68. Zhou X, von der Mark K, Henry S, Norton W, Adams H, de Crombrugghe $\mathrm{B}$. Chondrocytes transdifferentiate into osteoblasts in endochondral bone during development, postnatal growth and fracture healing in mice. PLoS Genet. 2014;10(12):e1004820.

69. Tsang KY, Chan D, Cheslett D, et al. Surviving endoplasmic reticulum stress is coupled to altered chondrocyte differentiation and function. PLoS Biol. 2007;5(3):e44.

70. Wang C, Tan Z, Niu B, et al. Inhibiting the integrated stress response pathway prevents aberrant chondrocyte differentiation thereby alleviating chondrodysplasia. Elife. 2018;7:e37673.

71. Voigt $P$, Tee $W W$, Reinberg $D$. A double take on bivalent promoters. Genes Dev. 2013;27(12):1318-1338.

72. Lui JC, Garrison $\mathrm{P}, \mathrm{Nguyen} \mathrm{Q}$, et al. EZH1 and EZH2 promote skeletal growth by repressing inhibitors of chondrocyte proliferation and hypertrophy. Nat Commun. 2016;7:13685.

73. Camilleri ET, Dudakovic A, Riester SM, et al. Loss of histone methyltransferase Ezh2 stimulates an osteogenic transcriptional program in chondrocytes but does not affect cartilage development. J Biol Chem. 2018;293(49):19001-19011.

74. Zhang F, Xu L, Xu L, et al. JMJD3 promotes chondrocyte proliferation and hypertrophy during endochondral bone formation in mice. J Mol Cell Biol. 2015;7(1):23-34.

75. Bradley EW, Carpio LR, van Wijnen AJ, McGee-Lawrence ME, Westendorf JJ. Histone deacetylases in bone development and skeletal disorders. Physiol Rev. 2015;95(4):1359-1381.

76. Vega RB, Matsuda K, Oh J, et al. Histone deacetylase 4 controls chondrocyte hypertrophy during skeletogenesis. Cell. 2004;119(4): 555-566.
77. Bonin CA, Lewallen EA, Baheti S, et al. Identification of differentially methylated regions in new genes associated with knee osteoarthritis. Gene. 2016;576(1 Pt 2):312-318.

78. Bradley EW, Carpio LR, McGee-Lawrence ME, et al. Phlpp1 facilitates post-traumatic osteoarthritis and is induced by inflammation and promoter demethylation in human osteoarthritis. Osteoarthr Cartil. 2016;24(6):1021-1028.

79. Singh $P$, Lessard SG, Mukherjee $P$, Rourke B, Otero M. Changes in DNA methylation accompany changes in gene expression during chondrocyte hypertrophic differentiation in vitro. Ann N Y Acad Sci. 2020. Epub ahead of print. https://doi.org/10.1111/nyas.14494.

80. Kim JK, Haselgrove JC, Shapiro IM. Measurement of metabolic events in the avian epiphyseal growth cartilage using a bioluminescence technique. J Histochem Cytochem. 1993;41(5):693-702.

81. Kudelko M, Sharma R, Cheah KS, Chan D. Comparison of proteomic datasets from hypertrophic chondrocytes in response to ER stress. Data Brief. 2016;7:449-451.

82. Chen J, Long F. mTORC1 signaling controls mammalian skeletal growth through stimulation of protein synthesis. Development. 2014;141(14):2848-2854.

83. Lee SY, Abel ED, Long F. Glucose metabolism induced by bmp signaling is essential for murine skeletal development. Nat Commun. 2018; 9(1):4831.

84. Cooper KL, Oh S, Sung Y, Dasari RR, Kirschner MW, Tabin CJ. Multiple phases of chondrocyte enlargement underlie differences in skeletal proportions. Nature. 2013;495(7441):375-378.

85. Wang J, Zhou J, Cheng CM, Kopchick JJ, Bondy CA. Evidence supporting dual, IGF-I-independent and IGF-I-dependent, roles for GH in promoting longitudinal bone growth. J Endocrinol. 2004;180(2):247-255.

86. Pennacchio LA, Ahituv N, Moses AM, et al. In vivo enhancer analysis of human conserved non-coding sequences. Nature. 2006;444 (7118):499-502.

87. Visel A, Akiyama JA, Shoukry M, Afzal V, Rubin EM, Pennacchio LA. Functional autonomy of distant-acting human enhancers. Genomics. 2009;93(6):509-513.

88. $\mathrm{Yu} M$, Ren B. The three-dimensional organization of mammalian genomes. Annu Rev Cell Dev Biol. 2017;33:265-289.

89. Moorthy SD, Davidson S, Shchuka VM, et al. Enhancers and superenhancers have an equivalent regulatory role in embryonic stem cells through regulation of single or multiple genes. Genome Res. 2017;27(2):246-258.

90. Furlong EEM, Levine M. Developmental enhancers and chromosome topology. Science. 2018;361(6409):1341-1345.

91. Osterwalder M, Barozzi I, Tissieres V, et al. Enhancer redundancy provides phenotypic robustness in mammalian development. Nature. 2018;554(7691):239-243.

92. Heinz S, Romanoski CE, Benner C, Glass CK. The selection and function of cell type-specific enhancers. Nat Rev Mol Cell Biol. 2015;16 (3):144-154. 


\section{DuEPublico}

Duisburg-Essen Publications online

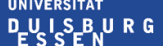

offen im Denken

ub universitäts bibliothek

This text is made available via DuEPublico, the institutional repository of the University of Duisburg-Essen. This version may eventually differ from another version distributed by a commercial publisher.

DOI: $\quad 10.1002 / \mathrm{jbmr} .4263$

URN: urn:nbn:de:hbz:464-20220214-114650-1

cc) (†) $\ominus$ This work may be used under a Creative Commons Attribution -

CC. NO ND NonCommercial - NoDerivatives 4.0 License (CC BY-NC-ND 4.0). 